\title{
Les risques d'épidémies de choléra liés à la commercialisation et à la consommation des produits maraichers crus à Ouagadougou : Facteurs déterminants et stratégies de réduction de risques.
}

\author{
Aline KAGAMBEGA \\ Département de Sociologie \\ Université Ouaga $1 \mathrm{Pr}$. Joseph KI-ERBO \\ kag aline@yahoo.fr
}

\section{Résumé}

La ville de Ouagadougou connait fréquemment l'épidémie du choléra. Pour connaître les risques d'épidémies du choléra, nous avons réalisé une étude qui s'est déroulée dans le périmètre maraîcher de Boulmiougou et le quartier de Pissy. L'objectif était de comprendre les facteurs de risques de contamination du choléra dans le circuit de la production, de la commercialisation et de la consommation des légumes crus. Au total, 55 entretiens individuels approfondis, des observations directes et non participantes ont été réalisés auprès des maraîchers, revendeuses, ménagères, traiteurs publics de légumes crus et consommateurs. Les risques de contamination du choléra sont liés à l'ignorance des modes de transmission de la maladie, des produits appropriés pour la désinfection des légumes crus et de l'impureté de l'eau d'arrosage des légumes compte tenu du faible niveau d'éducation, du manque d'argent pour l'achat de désinfectants adéquats et de la non-observation des règles d'hygiène. L'insalubrité des légumes crus est due en partie à l'insuffisance d'informations sur les effets de celle-ci. Ainsi, pour un quartier périphérique comme celui de Pissy, la sensibilisation, l'information de la population, ainsi que la formation des maraîchers de Boulmiougou apparait nécessaire pour une lutte efficace contre le choléra.

Mots clés : Epidémies de choléra, commercialisation, consommation, produits maraîchers crus, Ouagadougou, santé publique.

\begin{abstract}
The city of Ouagadougou frequently goes under the cholera epidemic.To assess the cholera epidemic risks, we have conducted a study on the vegetable gardening areas in Boulmiougou and Pissy. The objective was to understand the factors associated with cholera contamination in the production, in the marketing and in the consumption of uncooked vegetables chain. On the whole, 55 thorough individual interviews, direct and non-participating observations were conducted with vegetable growers, retail sale women, housewives, catering industry stakeholders and consumers of uncooked vegetables. Cholera contamination risks are related to the ignorance of the disease communication patterns, the non-use of appropriate products to disinfect uncooked vegetables and improper water used to grow vegetables due to the poor level of education of market gardeners, the lack of financial resources to buy appropriate disinfectants and the non-compliance with hygiene rules. Unhealthiness of uncooked vegetables is partly due to insufficient sensitization on its impacts. Therefore, for a peripheral area like Pissy, information and sensitization of populations as well as training of vegetable producers of Boulmiougou seem necessary for an effective fight against cholera.
\end{abstract}

Keywords: Cholera epidemics, marketing, consumption, uncooked vegetables, Ouagadougou, public health.

\section{Introduction}

L'urbanisation rapide pose de nouveaux enjeux en termes d'approvisionnement alimentaire et d'assainissement. L'Organisation des Nations Unies pour l'alimentation et l'agriculture (FAO, $2010: 2$ ) estimait que 925 millions d'individus souffrent de la faim et cette sous-alimentation touche essentiellement les continents Asiatique et Africain dont 239 millions en Afrique Subsaharienne. En plus, en février 2005 , on dénombrait dans le monde 36 pays qui étaient confrontés à de graves pénuries alimentaires. Jusqu'à 23 de ces pays se situaient en Afrique, contre sept en Asie, cinq en Amérique latine et un en Europe (FAO, 2005 : 131). 
Cette situation peut entraîner un accroissement des besoins alimentaires. Le Burkina Faso, pays soudano-sahélien, l'un des plus pauvres du monde, vit toutes ces situations.

Avec un taux d'urbanisation de $22,7 \%$, le nombre de citadins a quasiment doublé au cours des dix dernières années, passant de l,6 millions de personnes en 1996 à 3.181.967 millions en 2006 (Institut National de la Statistique et de la Démographie (INSD), Recensement Général de la Population et de l'Habitat (RGPH), 2006)). Cette population galopante entraîne une insécurité alimentaire. L'Enquête Multisectorielle Continue (EMC) de 2014 révélait qu'au Burkina Faso, 40,1\% de la population étaient pauvres (INSD, EMC, 2014 : 90). Selon I'INSD, EMC, (20I4 : 71 et 72$)$, sur une courte période de sept jours ayant précédé la collecte des données, environ un ménage burkinabè sur cinq avait fait face à une situation où il a manqué de la nourriture. Par ailleurs, $30 \%$ des ménages burkinabè avaient déclaré avoir manqué à un moment donné de la nourriture pour le ménage au cours des douze derniers mois.

Ce manque de nourriture a bouleversé la vie des agriculteurs et consommateurs car l'alimentation de la famille devenait une préoccupation essentielle. Sur le plan de la sécurité alimentaire, la culture maraîchère est un atout pour résorber ou aider à combler le déficit céréalier. Elle connaitra un bon développement avec les grandes sécheresses des années 1970 qui ont entraîné de mauvaises récoltes successives. Sur le plan national, les surfaces de production maraîchères varient entre 4000 et 6000 ha, selon les saisons. Les productions nationales réalisées étaient estimées à 75000 tonnes /an de produits maraîchers (Tougma, 2007 : 12).

La particularité de l'agriculture urbaine dans des villes comme Ouagadougou est l'utilisation des eaux usées pour l'arrosage des légumes. Ainsi, les sites de maraîchages sont créés généralement autour de ces eaux, plus ou moins stagnantes par des populations pauvres de zones périurbaines ou immigrantes des campagnes. Comme le disait Sadikou (2004), le principal danger des produits cultivés à l'aide des eaux usées repose sur la consommation de ces légumes. Cette pratique peut provoquer chez les consommateurs, des maladies diarrhéiques parmi lesquelles figure le choléra qui est une infection intestinale aiguë. C'est une maladie contagieuse épidémique due au Vibrio cholerae ou bacille virgule. La contamination est orale, d'origine fécale, par l'eau de boisson ou des aliments souillés'. Cette maladie invalidante sévit fréquemment et de manière irrégulière au Burkina Faso. De 197I à 1982, il y a eu 3.421 cas, dont 808 décès et de 1984 à 1995, il y a eu
4.012 cas dont 327 décès (Centre collaborant de l'Organisation Mondiale de la Santé (OMS), 200I).

Selon la Direction de l'hygiène publique et de l'éducation pour la santé du Ministère de la santé (2003: 8), le Burkina Faso a enregistré respectivement en 1991, 294 cas dont 33 décès ; en 1992, 33 cas ; en 1993, 36 cas ; en 1994, 8 cas ; en 1995, 540 cas dont 36 décès, en 1996, 188 cas dont 40 décès, en 1998, 1038 cas dont 52 décès, en 1999, 24 cas dont 3 décès, en 2001,544 cas dont 9 décès.

La situation épidémiologique en 2005 montre une persistance et a sévit principalement dans la capitale, notamment dans le quartier de Pissy. Un dénombrement des cas de choléra en 2005 par la Direction Régionale de santé du Centre a indiqué qu'il y a eu au total à Pissy, 602 cas de choléra, 89 cas à Kossodo, $5 \mathrm{I}$ cas à Paul VI, 249 cas au secteur 30 (cf : Comité National de lutte contre les épidémies/ Service de la surveillance épidémiologique du Ministère de la santé, 2005). Par ailleurs, Kassié (2007) en localisant en 2005 les principales zones de regroupement des cas de choléra par unité de $9 \mathrm{~km}^{2}$ dans un graphique, a montré que le nombre de cas est plus concentré au secteur 17 (49 cas, soit 5,4 cas $/ \mathrm{km}^{2}$ ), secteur dont fait partie Pissy contre ( 43 cas, soit $4,78 \mathrm{cas} / \mathrm{km}^{2}$ ) au secteur 23 , (40 cas, soit 4,44 $\mathrm{cas} / \mathrm{km}^{2}$ ) au secteur 18 et (38 cas, soit $4,22 \mathrm{cas} / \mathrm{km}^{2}$ ) aux secteurs 29 et 30 . L'auteur fait valoir qu'ailleurs, les cas semblent plus saupoudrés que concentrés alors que le secteur 17 a enregistré à lui seul près de 300 cas. Dans les secteurs 16, 18, 23, 28 et 30 , de 30 à 100 cas ont été enregistrés. Dans les autres secteurs de la ville, moins de 35 cas ont été identifiés. Le cas flagrant est que parmi les 402 cas de choléra résidant en zone urbaine, 179 résidaient au district sanitaire de Pissy, contre $|4|$ au secteur 30,64 à Kossodo et I8 à Paul VI (Kassié, 2007 : 48, 49 et 50). C'est ce qui justifie le fait que le choix des zones d'étude soit porté sur le quartier de Pissy et le site de maraîchage de Boulmiougou situé dans ce quartier. En plus du fait que ce site soit situé dans le quartier Pissy, ce qui augmenterait la probabilité de rencontrer plus d'enquêtés consommant les légumes du site de maraîchage de Boulmiougou, il est le seul site de maraîchage à Ouagadougou reconnu d'utilité publique par l'Etat burkinabè. Kaboré (2005) dans son étude soutenait que le site de Boulmiougou dispose d'une autorisation d'occupation délivrée par la mairie ; ce qui fait donc de ces maraîchers des interlocuteurs légaux de la municipalité, voire de l'Etat.

Face à cette situation, il est important de connaître les facteurs associés à l'ampleur de l'épidémie du choléra dans le quartier de Pissy (zone de consommation) et sur le site de maraîchage de Boulmiougou (zone de production) en vue de 
développer des stratégies adéquates de réduction des risques de contamination.

La question générale de recherche est la suivante :

Quels sont les problèmes de santé publique liés à la commercialisation et à la consommation des produits maraîchers crus dans la ville de Ouagadougou?

L'objectif visé par cette étude est d'analyser les risques d'épidémie de choléra liés à la commercialisation et à la consommation des produits maraîchers crus dans la ville de Ouagadougou.

Pour explorer l'objectif général, trois objectifs spécifiques ont été élaborés. Il s'agit :

I. D'analyser les pratiques à risques autour de la production, la commercialisation et la consommation des légumes crus chez les maraîchers de Boulmiougou et les habitants du quartier de Pissy ;

2. D'analyser les perceptions, les connaissances des acteurs de la production, la commercialisation et la consommation des légumes crus relatives à l'eau et à l'hygiène alimentaire ;

3. D'identifier avec les différents acteurs des solutions pour réduire les risques de contamination.

Après la partie introductive, nous traiterons de la revue de littérature, du cadre théorique et conceptuel, puis il sera question de la méthodologie, des résultats et discussion avant la conclusion.

\section{REVUE DE LITERRATURE}

\section{Fonction économique du maraîchage}

Une revue systématique de la littérature existante a permis de savoir que le maraîchage occupe une place importante dans l'économie du Burkina Faso. Selon la Direction Générale de la Promotion de l'Economie Rurale (DGPER, 2011: 34), la valeur totale des ventes des produits maraîchers était évaluée à 82 milliards de Francs de la Communauté Financière Africaine (F CFA).

Par ailleurs, il est ressorti dans l'étude de Ouédraogo (2004) que le maraîchage est l'un des rares secteurs de création de richesse. Traitant de la place et du rôle économique du jardinage dans la ville de Ziniaré, cet auteur soutenait que dans le contexte local de Ziniaré, l'activité maraîchère fournit à ceux qui s'y adonnent l'argent, l'un des signes les plus forts de la modernité dont les indices sont notamment l'électrification, le téléphone, le cinéma, le bar dancing. En effet, à la différence de l'agriculture traditionnelle destinée principalement à la subsistance, l'agriculture maraîchère de contre-saison vise principalement le marché, donc un revenu monétaire.

Aussi, cette culture maraîchère procure des revenus substantiels aux producteurs qui compensent le déficit céréalier. En effet, la pratique du maraîchage est une source supplémentaire de revenu monétaire pour les ménages au Burkina Faso. Il est mentionné dans l'étude de (Thiombiano, 2008: 34) que les ménages avaient tiré du maraîchage un revenu moyen de 77606 F CFA pour la campagne maraîchère de 2006 à 2007 et les maraîchers de la région du centre avaient le revenu moyen par tête le plus élevé (30206 F CFA/tête) pour la campagne maraîchère de 2005 à 2006.

En outre, une autre étude réalisée sur la citoyenneté et le foncier urbain à Ouagadougou a fait savoir que le Maraîchage Urbain et Péri-urbain (MUP) permet la création de revenus principaux ou complémentaires. C'est l'activité qui permet aux maraîchers de se réaliser, de se construire et de se penser «homme» dans la mesure où elle permet aux maraîchers de se soigner, de se nourrir et est leur seul espoir. Par le maraîchage, les maraîchers accèdent à une identité citadine, ce qui leur permet de satisfaire leurs besoins minimaux. Même si en terme économique le gain n'est pas identique pour tous les acteurs de cette activité, l'activité a, au moins le mérite de répondre à un objectif commun, la survie, tant individuelle que familiale. Les manœuvres ont un revenu mensuel qui leur permet de s'auto suffire. Le maraîchage apparait ainsi comme le grand organisateur qui leur assure subsistance, utilité et identité sociales. II se présente comme une réponse valable aux problèmes urbains tels que le manque d'emploi, la précarisation sociale, l'approvisionnement des villes. Particulièrement, à Boulmiougou, la moyenne en terme de revenu annuel est de 500.000 F CFA par maraîcher, multiplié par le nombre de maraîchers du périmètre, le site dégage en moyenne 128 millions de francs chaque année qui rentrent dans l'économie nationale (Kaboré, 2005).

\section{Risques de contamination des végétaux}

Une étude réalisée à Bobo-Dioulasso, s'est intéressée à l'utilisation des déchets urbains en maraîcheculture, notamment les risques de contamination des végétaux par les métaux lourds présents dans les déchets urbains. Cet auteur a estimé qu'il est nécessaire de déterminer les quantités de métaux lourds dans les sols et leur transfert dans les végétaux. Les résultats des analyses montrent que les sites étudiés étaient tous pollués par des métaux lourds ( $\mathrm{Pb}, \mathrm{Ni}, \mathrm{Zn}, \mathrm{Cu}, \mathrm{Cr}$ et $\mathrm{Cd}$ ). Le zinc et le plomb avaient les plus fortes teneurs et le site de Kodéni est le plus pollué en ces deux (2) éléments. Toutefois, en considérant globalement tous les métaux lourds dosés, le site de Kuinima est le plus pollué. Les teneurs totales de métaux lourds ( $\mathrm{Pb}, \mathrm{Ni}, \mathrm{Zn}, \mathrm{Cu}$ et $\mathrm{Cr}$ ) dosés dans la laitue varient peu d'un traitement à l'autre. Ces teneurs sont assez élevées pour certains métaux $(\mathrm{Pb}, \mathrm{Cu})$ et dépassent même les valeurs 
seuils. Parallèlement, la laitue prélevée en milieu paysan a aussi fait l'objet d'analyse. Les résultats donnent des teneurs en éléments traces métalliques (ETM) plus élevées comparativement à celles obtenues en phase de végétation. L'auteur conclut que les déchets urbains dans les jardins et champs contribuent à élever les risques de contamination des végétaux cultivés et sont de ce fait un danger pour la santé humaine (Bagbila, 2007). Aussi, Cissé (1997) a conduit sa thèse de doctorat à Ouagadougou sur la pollution bactériologique des eaux sur le site de l'Abattoir, du Canal central, de Boulmiougou et de Tanghin. II soutient que pour les eaux de rigole sur le site de l'Abattoir, les pollutions sont tout le temps audessus des normes sanitaires, le maximum allant jusqu'à $3.4 \mathrm{E}+07 \mathrm{CF} / 100 \mathrm{ml}$. Pour les eaux de bassin, les niveaux de pollution sont tout le temps au-dessus des normes sanitaires, le maximum allant jusqu'à 9.4 $\mathrm{E}+06 \mathrm{CF} / 100 \mathrm{ml}$. Pour les eaux du site de Canal central, les pollutions sont tout le temps au-dessus des normes sanitaires, le maximum allant jusqu'à 2.2 $\mathrm{E}+07 \mathrm{CF} / 100 \mathrm{ml}$. Pour les eaux de puits, les niveaux de pollution sont tout le temps au-dessus des normes sanitaires, le maximum allant jusqu'à $8.1 \mathrm{E}+05$ $\mathrm{CF} / 100 \mathrm{ml}$. Pour les eaux de barrage de Boulmiougou, le niveau de pollution le plus élevé est atteint en juin aussi bien en 1994 qu'en 1995. Le niveau le plus bas se situe au mois de février en 1994 et au mois de janvier en 1995. Les pollutions sont tout le temps au dessus des normes sanitaires, sauf en janvier et février aussi bien en 1994 qu'en 1995. Pour les eaux de puits, les niveaux de pollution sont en général au-dessus des normes sanitaires, sauf en janvier et février aussi bien en 1994 qu'en 1995. Quant aux eaux du barrage Tanghin, les pollutions sont tout le temps au dessus des normes sanitaires, sauf en janvier et février aussi bien en 1994 qu'en 1995, puis en décembre 1995. Pour les eaux de puits, les niveaux de pollution sont en général au-dessus des normes sanitaires, sauf en décembre 1994 puis en janvier et novembre 1995 (Cissé, 1997).

$\mathrm{Vu}$ l'importance du maraîchage et conscient des dangers de l'utilisation des eaux usées brutes pour l'arrosage des cultures, l'Etat burkinabé a mis en place une station d'épuration des eaux usées. Cette station est destinée à la collecte et au traitement des eaux usées de la ville de Ouagadougou. Pour ce qui est du cas spécifique des légumes, il est prévu que les maraîchers s'installent en aval de la station pour utiliser les eaux traitées. Mais, le problème demeure, car les autres sites de maraîchage ne sont pas dotés de cette infrastructure.

Selon nos enquêtes de terrain, d'autres stratégies de traitement des eaux usées ont lieu sur les sites de maraichage pendant les épidémies de choléra. Au cours de ces périodes, des équipes mobiles chargées de désinfecter et de traiter les points d'eau stagnante grâce souvent à l'appui du Ministère de la santé et des Organisations Non Gouvernementales (ONG) sont mises en place.

Cependant, force est de constater que malgré les nombreuses campagnes en faveur de l'assainissement et de la santé, la prise de conscience des populations pour l'assainissement n'est toujours pas assez suffisante, car des attitudes favorisant l'insalubrité des aliments continuent d'être adoptées, entraînant malheureusement la dégradation de la situation telle que la prolifération de certaines maladies.

\section{Influence potentielle de l'alimentation de rue sur la santé humaine}

Une autre revue de littérature a porté sur l'influence potentielle de l'alimentation de rue sur la santé humaine. Par rapport à cette thématique, une étude qui s'est attelée à l'Etude des facteurs influençant la précarité de l'hygiène des aliments de la rue dans la ville de Bobo-Dioulasso avait révélé que les vendeurs et consommateurs interrogés disposaient de très peu de connaissances en matière d'hygiène alimentaire. Parmi 18 vendeurs, 12 conservaient les produits avant la préparation à l'air libre, 7 vendeurs n'avaient pas une autorisation administrative de vente, 10 vendeurs ne connaissaient pas les maladies transmises par les aliments, 12 vendeurs affirmaient n'avoir jamais bénéficié de formation en matière d'hygiène. Parmi ceux qui avaient bénéficié d'une formation (06), 04 disent n'avoir pas reçu de formation depuis 2007. Quant aux consommateurs, 25 sur 54 , soit $46,29 \%$ ignoraient les maladies transmissibles par les aliments, 29 sur 54 consommateurs, soit $53,71 \%$ affirmaient connaître les maladies liées à l'hygiène alimentaire, 19 sur 54 consommateurs, soit $35,85 \%$ affirmaient avoir eu un malaise lié à la consommation des aliments vendus au marché.bLes douleurs abdominales représentaient $57,89 \%$ des cas de malaises (Diolompo, 2009).

Une étude précédente menée sur les caractéristiques de l'alimentation de rue dans cette même ville indiquait que pour 97,2\% (2 405 / 2 474) des consommateurs interrogés, la qualité des aliments de rue serait douteuse. Cette tendance a été confirmée lors des discussions de groupe (Drabo et al., 2008 : 38).

\section{Situations favorisant l'émergence et la diffusion de l'épidémie du choléra}

Une étude épidémiologique de la dynamique du choléra initié en République Démographique du Congo (RDC) avait pour but de comprendre les facteurs de récurrence épidémique et proposer des ajustements sur les approches opérationnelles. Les principaux résultats de ce travail de recherche ont 
permis de mettre en évidence qu'à peine $10 \%$ des zones de santé, toutes situées à proximité des lacs de l'est de la RDC, jouent le rôle de zones sanctuaires pour le choléra. Les résultats ont également permis de mettre en évidence la saisonnalité du choléra, avec moins de cas en saison sèche et le rôle spécifique des populations de pêcheurs, de commerçants, et de mineurs artisanaux dans l'émergence puis la diffusion des épidémies de choléra. II est aussi ressorti que certains foyers fonctionnant sur un mode métastable sont, depuis quelques années, passés à un fonctionnant endémique. C'est le cas des foyers de Kalemie et de Goma. Quatre groupes de facteurs avaient été étudiés pour analyser la dynamique du choléra à l'est de la RDC en général et essentiellement dans les zones lacustres. Parmi ces facteurs, il y a le rôle de la pluie, l'impact des urgences complexes et des catastrophes naturelles, l'impact des activités humaines liées à la pêche et au commerce de poisson et enfin le rôle des variations des blooms de plancton sur la dynamique temporelle du choléra (Bompangue Nkoko, 2009).

Au Burkina Faso, Kassié (2007) dans son étude menée dans la ville de Ouagadougou avait révélé que les secteurs périphériques étaient plus enclin à la propagation de la pandémie du choléra. Sur l'ensemble de la ville, le secteur 17 a été le premier à être atteint, suivi des secteurs 18 et 30 , puis 28 et enfin 23 dans une sorte de progression sélective au sein des secteurs périphériques.

La revue de littérature consultée fournit beaucoup d'informations sur la fonction économique du maraîchage, les risques de contamination par les végétaux, les situations favorisant l'émergence et la diffusion de l'épidémie du choléra, l'influence potentielle de l'alimentation de rue sur la santé humaine. Cependant, nous nous sommes rendu compte à travers nos lectures qu'il y a encore des pistes de recherche qui existent sur la thématique du maraîchage et de l'hygiène alimentaire. En effet, avant ce travail, aucune étude n'avait été conduite pour comprendre les facteurs liés aux risques de contamination du choléra dans le circuit de la production, de la commercialisation et de la consommation des légumes crus dans le site de maraîchage de Boulmiougou et le quartier de Pissy. II urge alors d'identifier les causes de cette pandémie dans les zones d'étude en vue d'en diffuser les résultats.

\section{CADRE THEORIQUE ET CONCEPTUEL}

Le cadrage théorique adopté dans cette investigation se rattache au paradigme épidémiologique. "L'examen de la littérature savante montre que le succès contemporain de la notion de " conduite à risque " repose largement sur l'application du paradigme épidémiologique à l'étude des comportements humains... lorsque ce paradigme est employé pour l'étude des comportements humains, il en résulte une prolifération des conduites à risque, ou plus précisément des conduites catégorisées comme telles »(Peretti-Watel, $2004: 103$ et 105). D'un point de vue heuristique, le paradigme épidémiologique offre un cadre plus approprié pour traiter le présent objet de recherche dans la mesure où il s'agit de mettre à nu les facteurs de risque des acteurs du maraichage pouvant favoriser la contamination du choléra dans le circuit de la production, de la commercialisation et de la consommation des légumes crus. Ignorer les modes de transmission de la maladie, des produits appropriés pour la désinfection des légumes, de l'impureté de l'eau utilisée pour la production des légumes est une conduite à risque pour la maladie du choléra.

Dans le cadre de l'étude, nous désignons par commercialisation, l'ensemble des opérations : cueillir, conserver, vendre.

Le risque est un danger éventuel plus ou moins prévisible.

Les consommateurs sont ceux qui consomment les légumes assaisonnés ou non assaisonnés dans les champs, dans les ménages, dans la rue et dans les restaurants.

Les produits maraichers crus sont les légumes susceptibles d'être consommés crus.

Le maraîcher est une personne qui cultive des légumes.

Des expressions en langue moore et dioula

" sâad-n-wùbdê wâ bâaga ", expression en moore, littéralement traduite en langue française

par: " la maladie qui fait à la fois déféquer et vomir ». Ce qui désigne la maladie du choléra.

"kabakuru », expression en dioula, littéralemente traduite en langue française par " pierre ». C'est le nom d'un savon traditionnel.

Ces deux expressions sont traduites par un concepteur de la Direction de la recherche des innovations en éducation non formelle et en alphabétisation (DRINA), ex Institut National d'Alphabétisation (INA).

\section{METHODOLOGIE}

Ouagadougou : caractéristique générale

Ouagadougou est la capitale politique du Burkina Faso située dans la province du Kadiogo et dans la région du Centre. Suite au redécoupage de la Commune de Ouagadougou en 12 Arrondissements 
et 55 secteurs consacré par la « loi 066-2099/AN du 22 décembre 2012 portant découpage des Communes urbaines à statut particulier au Burkina Faso ", il ressort que Ouagadougou compte I.915.102 habitants avec $49.60 \%$ de femmes et $50,40 \%$ d'hommes (Direction des Etudes et de la Planification (DEP), $2012: 6$ ).

L'étude est menée dans la ville de Ouagadougou, chef-lieu de la région du Centre où la culture maraîchère est assez développée et intensifiée. On y dénombre selon Tougma (2007: 2I), 2906.83 ha pour l'agriculture urbaine et 7821.58 ha pour l'agriculture périurbaine, soit un total de 10728.42 ha de terres agricoles pour l'Agriculture Urbaine et Périurbaine (AUP). Cissé (1997 : 99) a recensé 48 sites de maraîchage dans ladite ville. Les superficies totales sur ces sites varient d'environ 23 ha en août à 175 ha en décembre.

Les données utilisées ont été collectées à travers une revue documentaire et une enquête de terrain. Une grille d'observation et un guide d'entretien thématique ont été conçus et adaptés pour les différentes catégories d'informateurs.

Conformément aux objectifs de cette étude, l'entretien individuel approfondi a été utilisé comme technique principale pour recueillir l'information, selon une démarche socio-anthropologique. Ensuite, l'immersion progressive dans le milieu a consisté à aller discuter plusieurs fois avec les personnes concernées par l'étude. L'enquête est purement qualitative. Le séjour prolongé ou le nombre de jours passé sur le terrain a été très important car il a permis de croiser le maximum d'informations et surtout de parvenir à la saturation. La rigueur méthodologique n'a pas dépendu du nombre de personnes enquêtées, mais de l'enregistrement du maximum d'informations pertinentes et diversifiées. C'est ce que fait remarquer Olivier de Sardan (2005: 43) lorsqu'il souligne : "Chaque type d'enquête a ses formes de rigueur, c'est-à-dire ses formes spécifiques de validation ou de plausibilisation des données produites. Mais la rigueur de l'enquête de terrain n'est pas chiffrable, à la différence de la rigueur de l'enquête par questionnaire qui l'est en partie». De ce fait, pour l'échantillonnage, nous avons mis l'accent sur la diversité des enquêtés dans l'intention d'obtenir les avis des différentes catégories de personnes. Le nombre d'enquêtés a été déterminé à la fin de la recherche par le principe de la saturation.

Comme l'affirmait Quivy et Van Campenhoudt (1995: 184), l'entretien demeure l'outil le plus approprié pour recueillir ces types de données : " Ainsi, s'instaure en principe un véritable échange au cours duquel l'interlocuteur du chercheur exprime ses perceptions d'un évènement ou d'une situation, ses interprétations ou ses expériences tandis que par ses questions ouvertes, le chercheur facilite cette expression, évite qu'elle s'éloigne des objectifs de la recherche, et permet à son vis- à - vis d'accéder à un degré maximum d'authenticité et de profondeur».

Si le guide d'entretien a motivé notre choix, c'est qu'à la différence du questionnaire standardisé, le guide permet un processus itératif. Cela permettra de revoir notre approche du thème et de faire des feedback au cours de l'entretien.

La technique de la boule de neige a été utilisée pour produire les données, la technique de la triangulation pour croiser plusieurs sources d'information issues de tous les groupes stratégiques pouvant fournir des informations concernant les risques d'épidémie de choléra. Ces techniques ont été complétées par le principe de l'itération et de saturation d'information.

La technique de la boule de neige signifie que de chaque entretien est né de nouvelles pistes, de nouveaux interlocuteurs possibles, suggérés directement ou indirectement au cours de nos entretiens.

Cette technique a permis de choisir l'ensemble des participants à l'enquête de façon raisonnée, en ciblant par des entretiens informels les informateurs clés pouvant renseigner sur les centres d'intérêt explorés.

La technique de la triangulation signifie l'utilisation de plusieurs techniques, l'interrogation de plusieurs catégories de personnes, le croisement de plusieurs données provenant de plusieurs sources, la comparaison des données.

Cette triangulation des informations de personnes concernées par l'enquête (les acteurs du maraîchage, les revendeuses des produits maraîchers, les consommateurs) ainsi que des sources d'information (revue documentaire, entretiens individuels approfondis, les observations directe et non participante) a permis de confronter les différentes informations collectées afin d'éviter d'éventuel décalage.

La technique de l'itération a consisté à réécouter les entretiens ou à les retranscrire afin d'ajouter ou de reformer certaines questions dans le souci d'être conforme aux objectifs spécifiques de l'étude.

La technique de la saturation indique que le chercheur arrête de recueillir des informations lorsqu'il n'apprend plus rien de nouveau.

L'enquête s'est s'achevée après la saturation des informations. Contrairement à l'enquête par questionnaire, cette méthode n'inclut pas dans son dispositif un signal de " fin ».

Concernant les activités d'enquête de terrain, la première sortie a été une prise de contact avec le Délégué des maraîchers du site de maraîchage de 
Boulmiougou le 0I février 2009. Celui-ci par son intervention a pu convaincre les Maraîchers sur le bien-fondé de notre étude.

Les enquêtes se sont déroulées en trois phases. La première enquête s'est déroulée du 02 au 28 février 2009, mois correspondant à la période de grandes activités maraîchères à Ouagadougou (Tougma, 2007 : 38). Comme l'ont témoigné D'Arondel De Hayes et Traoré en 1990, la période de l'année la plus propice aux cultures maraîchères se situe pendant la saison sèche (Pangni, 2003 : 39). La deuxième enquête s'est tenue du 08 au 20 juin 2009 au niveau des services intervenant dans le domaine de l'hygiène avec les personnes ressources puis d'autres acteurs du maraîchage car nous avons estimé après analyse qu'il était nécessaire d'approfondir certains aspects. Nous avons fait une troisième enquête auprès d'autres personnes ressources en novembre et décembre 2015.

Au total, 55 entretiens individuels approfondis ont été réalisés. Le premier groupe d'enquêtés était composé de II Maraîchers, 10 vendeuses de légumes crus, 10 consommateurs, 9 ménagères, 2 restaurateurs, 4 traiteurs de légumes crus dans la rue. En plus de ce premier groupe de personnes interrogées, nous avons interviewé des personnes ressources composées d'un agent de la Ligue des Consommateurs du Burkina (LCB), du Laboratoire National de Santé Publique (LNSP), du Service d'Hygiène. Ces structures ont été retenues pour notre enquête parce que d'une manière ou d'une autre, chacune d'elle œuvre dans le domaine de l'Hygiène.

Le chef de service éducation pour la santé et l'assainissement (SESA) à la Direction Régionale de la santé des Hauts-Bassins, un Technicien d'Etat du Génie Sanitaire, chef de service de l'hygiène alimentaire et de l'éducation pour la santé, un autre Technicien d'Etat du Génie Sanitaire, agent au service de l'hygiène de Ouagadougou et un Ingénieur Agrochimiste, doctorant en Nutrition Humaine et Toxicologie Alimentaire à l'Université de Ouagadougou, Unité de Formation et de Recherche (UFR) en Science de la Vie et de la Terre (SVT), Centre de Recherche en Sciences Biologiques, Alimentaires et Nutritionnelles de l'Unité (CRSBAN) du Burkina Faso ont été par ailleurs interviewés en vue de savoir l'efficacité de certains produits utilisés comme désinfectant par les enquêtés.

Le représentant du Maire de l'Arrondissement de Boulmiougou est aussi interrogé car il est la première autorité de l'Arrondissement. En outre, un agent contrôleur d'enquête sur le site de Boulmiougou a été interrogé. C'est un agent du Ministère de l'Agriculture, de l'Hydraulique et des Ressources Halieutiques qui mène des enquêtes sur le site de maraîchage de Boulmiougou dans le cadre des activités maraîchères. II est chargé d'enseigner les bonnes pratiques dans la culture des légumes.

En recherchant l'avis de ces différentes catégories de personnes, nous recherchons un ensemble d'informations aussi complètes que possible, mais aussi une diversité de perspectives qui peuvent se renforcer, se compléter ou même se contredire.

Les enquêtes ont été possibles grâce aux avis favorables des différents responsables après une autorisation d'enquête. Les entretiens se sont déroulés la plupart du temps en langue moore et rarement en français. Les réponses des enquêtés ont été orales et enregistrées.

Pendant la période de l'enquête, nous avons fait des observations directes auprès des maraîchers de Boulmiougou, des revendeuses dans le marché de Pissy, des ménagères, des traiteurs publics de légumes crus et des consommateurs de ce quartier.

Le traitement des données a été fait à l'aide de l'outil informatique.

Concrètement, tous les entretiens formels intégralement enregistrés ont été transcrits à l'aide du logiciel de transcription numérique appelé "f4".

Ces entretiens transcrits ont été analysés à partir d'une classification thématique des extraits de discours.

Nous avons procédé ensuite à une analyse de contenu manuelle des discours des informateurs et une triangulation des données obtenues.

Une synthèse des différents thèmes dégagés en référence aux objectifs de cette phase d'enquête a été utilisée dans la présentation des résultats. En complément de cela, plusieurs extraits de discours significatifs ont été cités pour illustrer et interpréter.

\section{RESULTATS}

Les différents acteurs (producteurs, revendeuses, consommateurs des légumes crus) ont des pratiques à risques liés à leurs perceptions de l'eau utilisée, de la notion de saleté et de leur ignorance des modes de transmission de la maladie du choléra

Connaissances liées au mode de transmission de la maladie

Pour apprécier le niveau de connaissance des maraîchers, des revendeuses et des consommateurs sur les facteurs de risque de transmission du choléra, nous leur avons demandé de citer les modes de transmission du choléra. Pour les modes de transmission présumées, plusieurs tendances se dégagent. 
Une analyse des opinions a permis de constater que la majorité des personnes enquêtées ignore les déterminants du choléra. $A$ ce sujet, une ménagère s'exprime avec ironie : "Mais sachez que la saleté ne provoque pas le choléra, c'est une coïncidence. La personne devrait être malade et cela a coïncidé que la personne a mangé quelque chose et les gens pensent que c'est la nourriture. La saleté c'est de la vitamine (Rire)» (ménagère de 44 ans, gourounsi, mariée et non instruite).

Un grand nombre des personnes interviewées pense qu'il y a un lien entre les produits chimiques, le fumier et le choléra. C'est ce que stipule cette ménagère : "Nous désinfectons la laitue à cause $d u$ fumier qu'ils utilisent et des produits qu'ils utilisent pour pulvériser... C'est ce qui provoque le choléra» (ménagère de 45 ans, non instruite, mossi, mariée).

Les revendeuses de légumes crus ne dérogent pas à la règle. Une revendeuse pense que c'est le carbure qui provoque le choléra, raison pour laquelle, sa prévention réside dans l'interdiction du recours au carbure : "On met le carbure dans les fruits et légumes. C'est ce qui provoque le choléra. L'Etat doit interdire aux vendeurs de fruit et de légumes de mettre du carbure dans leurs légumes" (revendeuse de 70 ans, mossi, veuve, non instruite).

Si cette interviewée pense que c'est le carbure qui provoque le choléra, d'autres pensent plutôt à l'insuffisance de l'eau de lavage et d'arrosage des légumes. En témoigne cette revendeuse: "Les ménagères doivent bien laver les légumes, ceux qui arrosent aussi doivent bien arroser pour éviter la maladie du choléra, arroser avec suffisamment d'eau " (revendeuse de 23 ans, non instruite, mossi, mariée).

En outre, pour la plupart des maraîchers interrogé, le simple fait d'arroser fréquemment les légumes avec l'eau simple ou de les pulvériser peut éviter la bactérie du choléra : "Nous disons qu'aucune bactérie ne peut attaquer nos légumes parce que nous pulvérisons fréquemment et arrosons à tout moment et les légumes sont jolis à voir... Pour éviter la maladie du choléra, il faut que nous soyons plus propres qu'auparavant. Par exemple, au lieu d'arroser 2 fois par jour comme d'habitude, on passe à 3 fois. Au lieu de pulvériser les légumes une fois par semaine comme avant, pulvériser maintenant 2 à 3 fois pour que le médicament tue les bactéries et les microbes " (renchérit un maraîcher de 40 ans, niveau d'instruction (CM2), mossi, marié).

Certains maraîchers ont la conviction que même sans laver les légumes crus, on peut les consommer sans risques. A ce sujet, un interviewé déclare : "II est clair que nous mangeons les légumes crus sur le site. Dès que nous arrachons la tige de laitue, nous la consommons, il n'y a rien dedans. Quand on arrache la carotte aussi, on rince et on mange. Même si on n'a pas rincé, si ça n'a pas été pulvérisé récemment seulement, tu enlèves et tu manges. Qu'est-ce qui va se passer? Nous n'avons jamais appris aussi que quelqu'un est venu acheter des légumes ici consommer et suite à cela il est tombé malade, non, non. Nous n'avons jamais vu cela, nous n'avons jamais entendu non plus " (maraîcher de 62 ans, non instruit, mossi, marié).

\section{Connaissances liées à la désinfection des légumes crus}

L'analyse des déclarations faites par les enquêtés montre que la plupart d'entre eux ignore les produits de désinfection, le dosage, les techniques de lavage et le temps de désinfection. Par conséquent, elle utilise différents moyens ou produits pour la désinfection des légumes crus. Beaucoup de nos enquêtés utilisent des détergents tel que l'OMO, le savon, le bicarbonate, la cendre pour laver les légumes. Une ménagère se prononce à ce sujet : "Je lave la salade avec de l'OMO. Si tu laves avec l'OMO, si ce n'est pas Dieu qui a dit que tu vas contracter la maladie, en principe, il n'y a plus de danger " (ménagère de 55 ans, veuve, gourounsi).

Par ailleurs, plusieurs ménagères savent que c'est l'insalubrité qui provoque le choléra, mais ne savent pas qu'un simple lavage à l'eau, à l'OMO ou au savon ne tue pas la dite bactérie : "C'est l'insalubrité qui provoque le choléra. Lorsqu'on lave bien avec de l'OMO ou du savon pour consommer, il n'y a plus de risque ", affirme avec conviction une ménagère de 35 ans, mossi, célibataire.

Certains restaurateurs de légumes crus ne font pas exception. Un restaurateur rassuré de l'efficacité du bicarbonate pour la désinfection de la laitue s'explique en ces termes : "Quand c'est la laitue, nous lavons avec le bicarbonate. Voilà ! C'est comme cela que nous lavons " (restaurateur de 37 ans, niveau d'instruction CM2, mossi, marié).

Or, selon les dires de certains spécialistes du domaine, ces détergents détachent les saletés, permettent de décoller les germes qui sont collés aux légumes, mais ne les tuent pas. Ces produits généralement créent un milieu basique qui n'est pas favorable au développement des microorganismes. Ils créent un Potentiel Hydrogène $(\mathrm{PH})$ par exemple supérieur à 9 qui est un milieu défavorable pour leur vie. Ils peuvent détruire une bonne partie, mais ce n'est pas sûre que ces germes sont éliminés à 100\% (Cf. entretien avec un Ingénieur Agrochimiste Doctorant en Nutrition Humaine et Toxicologie Alimentaire à I'Université de Ouagadougou, UFRSVT, CRSBAN au Burkina Faso ; Un Technicien d'Etat du Génie Sanitaire, chef de service de l'hygiène alimentaire et de l'éducation pour la santé ; Un 
Technicien d'Etat du Génie Sanitaire, agent au service de l'hygiène de Ouagadougou ; Un chef de Service Education pour la Santé et l'Assainissement (SESA) à la Direction Régionale de la santé des Hauts-Bassins).

De même, un nombre important d'enquêtés avoue utiliser la glace, le citron, le vinaigre comme désinfectant. Une consommatrice utilisant la glace comme produit désinfectant assure : «Moi je mange fréquemment les carottes, mais avant de manger, j'achète la glace et je frotte longtemps la carotte à l'aide de la glace pour tuer les microbes et les bactéries... Je lave avec du savon, de la glace. On prend la glace pour frotter la carotte et ça enlève toute la saleté " (consommatrice de 22 ans, non instruite, célibataire). Ces produits tout comme les premiers suscités ralentissent le développement des microorganismes, mais ne les tuent pas. En effet, les microorganismes se développeraient favorablement dans l'intervalle de $\mathrm{PH}$ allant de 5 à 9 . Selon ces spécialistes, ces produits ont un $\mathrm{PH}$ inférieur à 5 , là aussi, c'est un milieu qui n'est pas favorable pour leur développement, mais ils subsistent (Cf. entretien avec un Ingénieur Agrochimiste Doctorant en Nutrition Humaine et Toxicologie Alimentaire à I'Université de Ouagadougou, UFR-SVT, CRSBAN au Burkina Faso ; Un Technicien d'Etat du Génie Sanitaire, chef de service de l'hygiène alimentaire et de l'éducation pour la santé ; Un Technicien d'Etat du Génie Sanitaire, agent au service de l'hygiène de Ouagadougou ; Un chef de Service Education pour la Santé et l'Assainissement (SESA) à la Direction Régionale de la santé des Hauts-Bassins).

Certains consommateurs rincent tout simplement les légumes et les assaisonnent.

Les revendeuses de légumes crus ne dérogent pas à la règle. En effet, une revendeuse qui pense qu'un lavage des légumes à l'eau simple peut tuer la bactérie du choléra argumente: "Les légumes se salissent au marché parce que quand un légume tombe, on prend et cela est source d'insalubrité. Quand on lave les légumes et que le sable sort, il ne peut plus avoir de microbe (bactérie) possible dans les légumes " (revendeuse de 45 ans, non instruite, mossi).

Pour certains profanes, l'eau du robinet peut être utilisée en lieu et place des désinfectants. En effet, quand on a l'eau potable, on n'a plus besoin de mettre l'eau de javel selon ces interlocuteurs : "Pour laver, on rince les autres légumes à l'eau simple une fois et la laitue deux fois. Pour bien laver, tu dois laver soit avec de l'eau de javel ou bien l'eau du robinet ", a lancé une ménagère de 44 ans, non instruite, gourounsi et mariée.

D'autres se contentent d'utiliser le désinfectant qui apparemment leur a donné satisfaction par manque d'information : "La plupart du temps, c'est l'eau de javel la croix qu'on utilise parce que ça, ça désinfecte mieux. A force d'utiliser, même à force d'utiliser les autres produits, on finit par opter pour la croix. Bon, surtout la laitue, il y a beaucoup de vers, donc, souvent quand on finit de laver, on les trouve déposés en bas. Donc, on trouve que ça tue bien. Voilà pourquoi j'ai opté pour l'eau de javel la croix», explique une restauratrice de 39 , de niveau d'instruction BEPC. En effet, chacun doit user de son expérience personnelle pour choisir le désinfectant qui selon son expérience est efficace.

C'est le même ressentiment pour cette traîteuse de légumes crus dans la rue qui affirme : " Je lave mes légumes avec du OMO ou avec l'eau de javel la croix. J'ai opté pour l'eau de javel la croix parce que j'utilise ça souvent pour laver des habits et sa enlève toutes les tâches " (traîteuse de légumes crus dans la rue ayant 30 ans, de niveau d'instruction primaire (CM2), gourounsi, mariée).

D'autres enquêtées font usage du sel pour la désinfection des légumes. Une revendeuse soutient : "Nous lavons la laitue avec de l'OMO et du sel " (revendeuse de 49 ans, bissa, non instruite). Or, il semble que le sel peut être utilisé pour conserver certains aliments compte tenu de son acidité, mais la quantité insignifiante utilisée pour désinfecter les légumes crus ne peut pas tuer la bactérie du choléra (Cf. entretien avec le chef de Service Education pour la Santé et l'Assainissement (SESA) à la Direction Régionale de la santé des Hauts-Bassins). Le sel a des pouvoirs osmotiques, il déshydrate les microbes (bactéries) par perte d'eau, mais n'est pas très efficace pour la désinfection des légumes crus (Cf. entretien avec un Ingénieur Agrochimiste Doctorant en Nutrition Humaine et Toxicologie Alimentaire à I'Université de Ouagadougou, UFR-SVT, CRSBAN Burkina Faso).

Des arguments avancés prouvent qu'il y a des enquêtées qui connaissent les désinfectants appropriés, mais ignorent le dosage, les techniques de lavage et le temps de désinfection.

Différents moyens sont utilisés par les personnes interviewées pour laver les légumes crus. Ces différences se perçoivent au niveau du type de désinfectant utilisé et du temps de désinfection. La durée de désinfection va d'une minute à une heure de temps et varie d'un traiteur à un autre selon la disponibilité et le jugement de tout un chacun : "Nous mesurons avec le capuchon. Nous avons un petit capuchon, le capuchon d'un petit bidon que nous utilisons pour mesurer trois fois la quantité $d u$ capuchon... Souvent nous mesurons une ou deux fois en fonction de la quantité de la laitue " (restaurateur ayant 37 ans, niveau d'instruction CM2 parlant du bicarbonate en liquide). 
A une autre restauratrice d'ajouter : "La plupart du temps, on lave la laitue trois fois, premièrement avec de l'eau simple, après avec l'eau de javel et la troisième fois, on rince. Quand on plonge la laitue dans l'eau de javel, on n'enlève pas sur le champ. On laisse ça un peu, un peu de temps, disons 10 à $15 \mathrm{mn}$ avant d'enlever ", argumente la restauratrice de 39 ans, de niveau d'instruction BEPC. Alors que dans les normes, on doit tremper les légumes crus dans la solution d'eau de javel préparée où ( $I$ part de javel correspond à II, 5 parts d'eau) pendant $30 \mathrm{mn}$ pour l'eau de javel de $8^{\circ}$ et ( 1 part de javel correspond à 19 parts d'eau) pendant $30 \mathrm{mn}$ pour l'eau de javel de $12^{\circ}$ (Direction de l'hygiène publique et de l'éducation pour la santé du ministère de la santé, 2003 : 26).

Toujours dans le même ordre d'idées, une traîteuse de légumes crus dans la rue après avoir dissout les comprimés de permanganate dans l'eau, incline le bidon pour mettre un peu de son contenu dans un récipient contenant de l'eau et désinfecte ses légumes: "Nous inclinons le bidon pour mettre une quantité dans l'eau. Cela dépend de la quantité de la laitue. La laitue peut être d'une petite quantité, de fois aussi, la laitue est d'une quantité importante. On met le liquide en fonction de la quantité de la laitue. Voilà ! (traiteuse de légumes crus dans la rue âgée $3 \mathrm{I}$ ans, mossi, mariée, niveau d'instruction CE2).

Une ménagère de niveau d'instruction supérieur avoue qu'elle n'a aucune idée par rapport à la désinfection des légumes crus : "On regarde à peu près la quantité de la laitue. On mesure avec le capuchon du bidon peut être trois fois comme ça pour que l'odeur aussi ne soit pas trop, sinon il n'y a pas de mesure entant que telle. On regarde à peu près la quantité de la laitue et puis on essaie de doser quoi » (BAC + 4, 33 ans, mossi, mariée).

Une consommatrice de niveau d'instruction supérieur affirme elle aussi qu'elle n'a aucune notion en ce qui concerne le dosage des désinfectants : «... ça, je ne connais pas, je fais comme ça seulement. Comme je ne connais pas la chimie, je ne peux pas mesurer pour savoir quelle quantité peut tuer les microbes (bactéries) ou pas" (consommatrice de niveau d'instruction supérieur (BAC +5 , gourounsi, célibataire, 30 ans).

En résumé, retenons que la totalité de nos enquêtés ne lave pas convenablement les légumes crus d'une manière ou d'une autre, soit par ignorance du désinfectant susceptible de désinfecter les légumes crus, soit par ignorance du temps de désinfection. Force est de reconnaître que les populations du site de maraîchage de Boulmiougou et du quartier de Pissy ne sont pas assez sensibilisées et qu'il n'y a pas suffisamment de réglementation en ce qui concerne les normes en matière de consommation des légumes crus.

Connaissances liées aux légumes crus devant être désinfectés

Concernant les connaissances liées aux légumes crus devant être désinfectés, les avis des enquêtés sont partagés. La majorité des personnes interviewées pense que c'est la laitue seulement qui doit être désinfectée pour de multiples raisons. Nous en voulons pour preuve, l'intervention d'une ménagère qui réduisant l'insalubrité des légumes crus au sable, s'expliquait en ces termes : "On lave la laitue avec l'eau de javel parce que les feuilles de laitue contiennent du sable. Ce qui n'est pas le cas dans l'oignon" (ménagère âgée de 58 ans, mariée et non instruite). Un consommateur partageant ce point de vue soutient : "La carotte et l'oignon ne sont pas sales car ils sont enterrés. On n'est pas tenu de les désinfecter avant consommation " (consommateur de niveau d'instruction primaire (CEI), 22 ans, mossi).

Pour une consommatrice, le piment de l'oignon ne peut permettre à une bactérie d'y vivre : " L'oignon est pimenté et ni un microbe, ni une bactérie ne peut être dans l'oignon. C'est pourquoi l'oignon ne doit pas être désinfecté " (économiste âgée de 28 ans, catholique d'ethnie moaga).

Les traiteurs de légumes crus eux-aussi n'ont aucune notion des légumes crus devant être désinfectés : "La laitue se lave avec le permanganate. Les autres légumes se lavent avec $d u$ savon (kabakuru ou SN-Citec). La laitue peut contenir des chenilles, alors qu'il n'en est pas de même pour les autres légumes qui peuvent être pelés». Fait savoir un préparateur de salade dans la rue, âgé de 38 ans, de niveau d'instruction secondaire (classe de quatrième).

Perception relative à l'eau utilisée pour le maraîchage Pour la réalisation de leurs activités, les maraîchers utilisent diverses sources d'eau pour l'irrigation des cultures. Au niveau du site de maraîchage de Boulmiougou, deux types d'eaux sont utilisés ; l'eau des puits et l'eau du barrage. Selon les dires des maraîchers enquêtés, la proportion des maraîchers qui utilisent l'eau des puits est nettement plus élevée que ceux qui utilisent l'eau du barrage. Sur onze maraîchers interrogés, huit sont ceux qui ont déclaré utiliser l'eau des puits. 
L'eau du forage (pompe) n'est pas utilisée pour arroser les légumes pour deux raisons.

La plupart (6/8) de ceux qui sont éloignés du forage trouve qu'il est insensé de laisser l'eau du barrage ou d'un puits à côté de soi et de se déplacer un peu plus loin pour chercher l'eau du forage. C'est ce que confie un des maraîchers : "Si nous laissons l'eau à côté de nous pour aller au niveau du forage, est-ce qu'ils (les gens) ne vont pas nous traiter de fou ?" (maraîcher de 18 ans, non instruit, mossi et célibataire).

Cette vision de la plupart des maraîchers enquêtés est due au fait que les maraîchers ne pensent pas que l'eau du forage a un avantage quelconque comparativement à l'eau du barrage et du puits qui sont sans doute souillées. Ainsi, certains maraîchers $(3 / \mathrm{II})$ sont proches du forage et n'arrosent pas avec l'eau du forage parce qu'ils trouvent que celui-ci est difficile à pomper. Ces maraîchers sont minoritaires comparativement à d'autres $(6 / \mathrm{II})$ qui refusent catégoriquement d'arroser les légumes avec l'eau du forage non pas parce qu'ils estiment que le forage est situé loin d'eux ou qu'il est difficile à pomper, mais parce que ces derniers jugent que l'eau du puits ou du barrage fait développer mieux les légumes. A ce sujet, un maraîcher s'explique en ces termes : "On ne peut pas enlever l'eau du forage pour arroser les légumes parce qu'à mon avis, cette eau n'est pas très bonne comme l'eau des puits, parce que déjà quand tu enlèves l'eau du forage pour boire, tu sais déjà ce qui est dedans et ce n'est pas bon. Le goût même de l'eau n'est pas bon. Donc, si on utilise l'eau de la pompe pour arroser certains légumes et l'eau des puits pour arroser d'autres légumes, on se rend compte que l'eau du puits est mieux adaptée pour l'arrosage des légumes. Les légumes qui sont arrosés avec l'eau du puits se développent mieux par rapport aux autres légumes arrosés avec l'eau du forage» (maraîcher de 62 ans, non instruit également, mossi et marié).

Un autre maraîcher renchérit : "Si nous prenons l'exemple du site de maraîchage de Tanghin, (les déchets biomédicaux du CHU YO et d'autres eaux insalubres sont déversés dans un barrage situé non loin de l'Hôpital et c'est cette eau du barrage qui est utilisée pour l'arrosage des légumes de ce site), vous savez que ce n'est pas propre comme ce site. Mais quand vous regardez les légumes qu'ils cultivent, leurs légumes se développent mieux par rapport aux nôtres. C'est parce que chez eux, il y a plus de saleté que chez nous. Pour que les légumes réussissent, il faut que les lieux où on cultive les légumes soient sales) (maraîcher de 25 ans, non instruit, mossi et marié).
Ainsi, les eaux de puits et de barrage sont utilisées par les maraîchers pour l'arrosage et le lavage des légumes crus avec tous les risques de pollution.

Cependant, les conceptions des maraîchers ne reflètent pas une prise de conscience de cette pollution. Sur II maraîchers interrogés, aucun d'entre eux ne fait un lien entre l'eau des puits et du barrage de Boulmiougou d'avec la maladie du choléra. Un maraîcher argumente en ces termes : "Pendant l'épidémie du choléra, les agents du service d'hygiène étaient venus mettre des produits dans nos puits pour tuer les bactéries du choléra. Si on refusait, ils allaient refuser à leur tour d'acheter nos légumes. C'est pour cette raison que nous avons accepté sinon, il n'y a pas de bactérie de choléra dans nos puits» (maraîcher de 25 ans, non instruit, mossi, marié).

Cette conception est partagée par presque tous les maraîchers du site de maraîchage de Boulmiougou interrogés. Le délégué général des maraîchers du site de Boulmiougou fait savoir expressément : "On arrose avec l'eau de puits. Quand nous récoltons les légumes, nous les lavons avec de l'eau des puits avant de les vendre. Nous mangeons les carottes et concombres dans les champs, mais nous n'avons jamais eu mal quelque part. Si une maladie vient, c'est la maladie qui est venue (rire générale). Si ceux qui sont au marché n'entretiennent pas les légumes, ça peut se salir. Dans le site de production, nous ne lavons pas avec de l'eau sale, c'est l'eau du puits que nous utilisons pour laven» (maraîcher âgé de 66 ans, mossi, marié et illettré).

La perception des exploitants concernant la potabilité de l'eau est partagée par certaines revendeuses et ménagères. Dans ce sens, une revendeuse de légumes crus argumente : "Les légumes sont propres étant donné que les maraîchers arrosent avec de l'eau propre, l'eau du barrage" (revendeuse de légumes crus, âgé de 45 ans, non instruite, mossi).

Par ailleurs, une ménagère qui partage cette même opinion défend : "Nous lavons la laitue avec l'eau du puits. L'eau du puits est propre. Quand tu vois l'eau de certains puits, c'est comme l'eau du robinet, tellement c'est claire " (ménagère de 30 ans, mossi, mariée, non instruite). A l'image de cette ménagère, beaucoup d'interviewés pensent que quand l'eau est claire, elle est limpide et est sans risque de contamination.

Cependant, si les enquêtés sont unanimes que l'eau du puits est potable et ne présente aucun risque pour la consommation, certains d'entre eux sont réservés quant à l'eau du barrage. La perception des informateurs est mitigée à ce niveau. Toutefois, une majorité pense qu'elle est insalubre, en témoigne cette revendeuse : "L'eau du puits est plus propre 
que l'eau du barrage parce que l'eau provient du sous-sol. En ce qui concerne l'eau du barrage, l'eau de ruissellement vient de partout et se déversent dedans. Lorsqu'il pleut, même l'eau des WC (toilettes intimes) rentre dedans. Pourtant, en ce qui concerne le puits, ça, ça provient du sous-sol. L'eau de ruissellement n'entre pas dedans " (revendeuse de 23 ans, non instruite, mossi et mariée).

Bien que reconnaissant la mauvaise qualité de l'eau du barrage quand l'eau tarit, des intervenants déclarent être obligés de l'utiliser car elle permet de pallier souvent le manque d'eau : «... Quand l'eau du barrage aussi devient propre, vous pouvez l'enlever et arroser avec. Mais lorsque l'eau tarit et que les gens rentrent pour pêcher le poisson, nous n'arrosons plus avec l'eau du barrage. Nous attendons qu'il pleuve pour que l'eau qui était sale coule avant de commencer à arroser encore avec l'eau du barrage. Au moment où tout le monde rentre pour pêcher le poisson, si ce n'est pas que nous n'avons pas vraiment le choix, nous cessons d'utiliser l'eau du barrage pour arroser les légumes " soutient un maraîcher de 42 ans, de niveau d'instruction primaire (CPI), marié, mossi qui arrose généralement ses légumes avec l'eau du puits.

D'autres exploitants arrosent leurs légumes avec l'eau du barrage mais ne l'avouent pas. En effet, après avoir réalisé un entretien avec un maraîcher qui soutenait qu'il arrose ses légumes avec l'eau de puits, nous l'avons vu plusieurs fois dans les jours qui suivaient arroser ses légumes avec l'eau du barrage, alors que cette information n'avait pas été avouée au cours de l'entretien. Cette malhonnêteté est due à la confiance que ces maraîchers ont de l'eau du puits comparativement à l'eau du barrage.

En somme, les eaux utilisées par les maraîchers pour arroser les légumes crus sont de mauvaises qualités, on y trouve sans doute, des bactéries susceptibles de transmettre la maladie du choléra.

\section{Perception sociale de la notion de saleté}

Une analyse des opinions et évocations des interviewés permet de constater que les risques de contamination dépendent de la manière dont les enquêtés perçoivent la notion de saleté. Pour un grand nombre d'interviewés, la saleté se résume à tout ce qui est visible (le sable, la boue). C'est ce que révèlent des discours relatés : "Les légumes ne peuvent pas se salir au marché. Les légumes tant qu'ils ne sont pas pourris, ne sont pas susceptibles d'être atteints par la bactérie du choléra. Quand par exemple la tomate est pourrie, la poussière peut pénétrer. Mais quand on lave, la poussière quitte», précise une ménagère âgée de 20 ans, de niveau $d^{\prime}$ instruction secondaire $\left(3^{\mathrm{eme}}\right)$, mariée, gourounsi.
Un consommateur réduisant lui aussi l'insalubrité à la poussière et au sable défend : "On n'a pas besoin de laver les autres légumes autre que la laitue avec du permanganate parce que la peau est lisse. La tomate est lisse et quand on rince, il ne peut plus avoir de saleté possible " (consommateur ayant 25 ans, célibataire et de niveau d'instruction secondaire $\left(3^{\text {ème }}\right)$ ).

C'est également le point de vue de cette revendeuse qui renchérit : "... Regarde, nous ne disposons pas de table ici, mais nous avons superposé des sacs pour ne pas salir les légumes. Même s'il pleut, l'eau sale ne peut pas être en contact avec les légumes. Les mouches selon moi ne peuvent rien faire aux légumes. C'est quelque chose de sec. Les mouches n'y peuvent rien. Ce n'est pas une nourriture fraîche comme des galettes. C'est la poussière seulement qui peut salir les légumes mais là aussi, nous lavons " (revendeuse âgée de 70 ans, veuve, illettrée et mossi).

A cause des risques de contamination par la production, la commercialisation, les modes de traitement des légumes crus avant consommation, la consommation de ces légumes crus peut être une source de maladie pour les consommateurs.

Les pratiques à risques des consommateurs sont déterminées par le faible niveau d'éducation de la population, leur situation socio-économique et l'inobservation des règles d'hygiène susceptibles de transmettre la bactérie du choléra.

Risques de contaminations liés au faible niveau d'éducation de la population enquêtée

Les enquêtes de terrain ont révélé qu'il y a un lien entre le niveau d'instruction et les pratiques des acteurs concernant la propreté des légumes crus. En effet, même si les normes au niveau des pratiques de désinfection ne sont pas respectées, parmi les quinze préparateurs de légumes crus interrogées (Ménagères, restaurateurs et traiteurs de légumes crus dans la rue) dont six instruits et neuf illettrés, toutes les personnes instruites (6/6) désinfectent au moins la laitue avec le permanganate ou l'eau de javel. Ce qui signifie que même si les doses de ces désinfectants ne sont pas connues par ces enquêtés scolarisés, ces derniers savent au moins quel désinfectant utiliser pour désinfecter les légumes crus dans le but de prévenir la maladie du choléra. Ainsi, les enquêtés qui désinfectent les légumes plus ou moins convenablement avec l'eau de javel ou du permanganate sont majoritairement instruits. L'intervention de cette consommatrice ayant 42 ans, de niveau d'instruction supérieure (DEUG II) est illustrative de cet état de fait : "Je lave tous les 
légumes au même titre que la laitue... L'eau de javel est efficace à $100 \%$ ".

Parmi les neuf enquêtés non scolarisés interviewés, $(2 / 9)$ seulement avouent laver de temps en temps la laitue soit avec l'eau de javel ou du permanganate.

Alors, nous pouvons affirmer que l'instruction a un impact sur le comportement des acteurs en matière de pratique de propreté des légumes car il existe un lien entre le type de désinfectant utilisé et l'instruction. Plus l'on est instruit, plus on a recourt aux désinfectants appropriés pour la désinfection les légumes crus. Ainsi, l'instruction donnerait aux individus la capacité d'appréhender les conséquences de consommer des légumes crus insalubres. La majeure partie des individus interrogés dans cette étude, qui ont un faible niveau d'instruction ou qui sont sans niveau, adoptent plus de pratiques à risque. Cela s'expliquerait en partie par le fait qu'il n'y a pas suffisamment d'action entreprise pour promouvoir la santé des consommateurs des légumes crus du site de maraîchage de Boulmiougou et du quartier de Pissy.

Cependant, si le niveau d'instruction a une influence sur les pratiques des consommateurs en matière d'hygiène des légumes crus, il n'en est pas de même pour les autres indicateurs tels que le sexe, l'âge, l'ethnie, la situation matrimoniale et la fonction de l'enquêté. En effet, nous nous sommes rendu compte au cours de nos enquêtes que le niveau de pratiques de salubrité des légumes crus ne dépend nullement du sexe, de l'âge, de l'ethnie, de la fonction ou de la situation matrimoniale. Selon que l'enquêté soit homme ou femme, jeune ou vieux, mooaga ou gourounsi, ménagère ou salarié, marié ou pas, nous n'avons pas pu constater des divergences dans leurs pratiques de propreté.

Risques de contaminations liés à la situation économique de la population enquêtée

Lors des entretiens individuels approfondis, le niveau du pouvoir d'achat du consommateur était déterminant pour la consommation de légumes crus salubres. La quasi-totalité de nos enquêtés a décelé un seul facteur le plus souvent pour expliquer la consommation de légumes crus insalubres. II s'agit de la pauvreté qui a été développée par plusieurs enquêtés pour justifier l'achat de légumes crus pourris. En effet, les légumes pourris sont vendus moins chers, ce qui incite certaines personnes interrogés à les acheter. C'est ce que traduit cette revendeuse: "Il y a des gens qui préfèrent ce qui est pourri. Ces tas coûtent $200 \mathrm{~F}$, alors que ce qui est pourri coûte $50 \mathrm{~F}$ (la même quantité de tomate). Ceux dont l'argent de la popote n'est pas suffisant achètent ces légumes. Chacun achète selon ses moyens " (revendeuse de légumes de 70 ans, mossi, veuve et illettrée).

Ainsi, par manque de moyens économiques, certains clients se sentent obligés d'acheter des légumes pourris. Nos observations concordent avec certaines informations recueillies lors des entretiens. Nous avons effectivement observé plusieurs clients se ruer sur des tas de tomates pourris pendant qu'à côté, il y avait encore de jolis tas de tomates, mais chers par rapport à ce qu'ils ont achetés.

Cette tendance s'explique par le fait que les populations généralement pauvres, peu ou pas instruites accordent peu d'intérêt à l'hygiène, l'essentiel pour elles étant de survivre. En témoigne cette revendeuse de légumes crus : " Je lave la laitue avec du savon. Je lave avec n'importe quel savon que je trouve "kabakuru " etc... J'ai déjà fait le choléra. On dit c'est le permanganate qui tue la bactérie, mais comme je n'ai pas l'argent pour acheter le permanganate, je pense qu'à défaut $d u$ permanganate, il est mieux de laver avec du savon " (revendeuse de légumes crus, âgé de 44 ans, gourounsi, mariée).

A une autre de renchérir : "Pour laver la laitue, je fais mousser l'eau à l'aide du savon "kabakuru » pour immerger la laitue. Je n'ai pas l'argent pour acheter les autres désinfectants. II y a un de mes fils qui a dit à ses enfants de ne pas consommer. Je me suis tu. Entre temps, j'ai vu ses enfants et sa femme consommer. J'ai continué à me taire. Je ne suis pas morte. Est-ce que lui (son fils), il a de l'argents (revendeuse de légumes de 70 ans, mossi, veuve et illettrée).

Ainsi, bien que reconnaissant l'inefficacité de certains produits dans la désinfection des légumes crus, des intervenants déclarent être obligés de les utiliser parce qu'ils ne disposent pas d'argent pour acheter les désinfectants habilités à jouer ce rôle.

Toujours pour parler de la pauvreté qui est un frein à la consommation de légumes crus salubres, un maraîcher réclame : "II faut que les autorités aident les gens à sortir de la misère. Le choléra a un lien avec la pauvreté. Si tu deviens riche, tu vas faire attention aux nourritures. Par contre, si tu es assis et tu ne peux pas avoir à manger, si tu vois une nourriture, tu t'acharnes dessus seulement, tu ne vas même pas t'arrêter laver ta main. Il faut que l'Etat fasse l'effort pour que les gens sortent de la misère... Si toi aussi tu es heureux, tu vas te méfier. Si je fini par exemple de cultiver, je vais me rendre propre comme un fonctionnaire, m'asseoir bien manger avant de continuer mon travail... Le fait de cueillir un concombre manger en même temps en arrosant là est dû à la famine. Si tu manges et que tu es rassasié, tu ne vas pas cueillir un concombre ou couper une feuille de laitue manger pendant que tu arroses" (un 
maraîcher âgé de 50 ans, de niveau d'instruction primaire (classe de CEI), mossi et marié).

Risques de contaminations liés à l'inobservation des règles d'hygiène

Hormis les comportements à risque liés au faible niveau d'éducation de la population enquêtée, aux problèmes de ressources économiques, on remarque d'autres comportements à risque liés à l'inobservation des règles d'hygiène.

Sur ce plan, une consommatrice, sociologue ayant fait rétention de son âge déclare : "Souvent, il m'arrive de descendre de ma moto, prendre une carotte pour consommer sans laver ma main avec toute la distance que j'ai parcourue».

Dans le même ordre d'idées, un exploitant maraîcher avoue : "Nous assaisonnons la salade ici souvent pour consommer en groupe. Nous ne nous fatiguons pas pour désinfecter les légumes avec l'eau de javel et autres. Souvent à midi, nous découpons la laitue, les tomates, les carottes, les concombres et autres, assaisonner, manger sur le site, mais nous n'avons jamais entendu qu'après avoir mangé la salade, un maraîcher est tombé malade " (un exploitant maraîcher de niveau d'instruction primaire (CPI), marié, mossi et ayant 42 ans).

Par ailleurs, la quasi-totalité de notre population cible (maraîchers, revendeuses, consommateurs) interrogée (45/46) estime avoir déjà consommé la carotte ou le concombre au moins une fois sans que celle-ci ne soit désinfectée au cours des cinq dernières années.

En outre, selon les dires des personnes interrogées, dans la plupart des ménages (6/9), le plat de salade est le plus souvent servit pour plusieurs personnes qui le mangent en groupe en rinçant tout simplement leurs mains. Les maraîchers ne font pas exception. En effet, lors de nos visites sur le site de maraîchage de Boulmiougou, nous avons rencontré plusieurs fois des maraîchers consommer de la salade en commun sur le site sans désinfecter les légumes crus et sans laver leurs mains au savon. Ces comportements peuvent être des sources de transmission du choléra. Or, l'utilisation du savon est une des mesures à prendre pour éviter les risques de contamination du choléra.

De même, la majorité de nos enquêtés, que ce soit au niveau des traiteurs de légumes crus dans la rue ou dans les restaurants n'ont pas une autorisation administrative de vente.

Par ailleurs, parmi les quatre traiteurs de légumes crus interrogés dans la rue, aucun d'entre eux ne portait ni un bonnet, ni un tablier lors de leur service.

Aussi, parmi les deux restaurateurs interrogés, un d'entre eux avait porté un tablier et aucun d'entre eux n'avait porté de bonnet lors de notre visite.
En plus, l'eau où les traiteurs de légumes crus lavent les ustensiles de cuisine n'est parfois pas propre. Nous avons pu constater chez un traiteur de légumes crus dans la rue à Pissy que tous les clients qui désiraient laver leurs mains avant de manger les lavaient dans l'eau utilisée pour laver les ustensiles. En effet, un employé de ce traiteur de légumes crus a dit à une femme qui plongeait sa main droite dans l'eau utilisée pour le lavage des assiettes afin de prélever un peu d'eau laver ses deux mains hors du plat, de plonger toutes ses deux mains dans le plat pour laver car selon l'employé, le fait de prélever une quantité d'eau à la main pour laver les mains hors du plat contribuait à mouiller l'environnement immédiat.

En somme, le manque de moyen économique, le faible niveau d'éducation de la population et la non observation des règles élémentaires d'hygiène amène plusieurs de nos enquêtés à adopter des comportements peu recommandés susceptibles de provoquer la contamination par la bactérie du choléra. Ces pratiques non hygiéniques font croire qu'il y a des possibilités inquiétantes de propagation du Vibrio cholerea aux consommateurs de légumes crus. En effet, il y a une influence des conceptions erronées et des pratiques inadéquates sur les risques élevés de contamination de la maladie du choléra chez les maraîchers, revendeuses, traiteurs et consommateurs des légumes crus. Même si certains acteurs interviewés dans la présente étude reconnaissent l'existence de risques pour la santé, cette conscience ne prédispose pas à des attitudes de prévention chez tous les enquêtés. Sur les sites de ventes, ce qui est qualifié de "propre »par le consommateur n'est pas forcément exempt de germe, tout comme le "sale " n'est pas basé sur un critère objectif de jugement parce que «la propreté ou la saleté absolue n'existe pas, sinon aux yeux de l'observateur" (Drabo et al., 2008 : 39).

\section{Activités menées par les institutions dans le cadre de la lutte contre l'épidémie de cholera}

Les entretiens avec les personnes ressources ont révélé qu'il n'y a pas assez d'activités de formation, d'information et de sensibilisation menées sur l'hygiène des légumes crus dans le site de maraîchage de Boulmiougou. A l'issue de l'entretien fait avec l'agent technique d'agriculture spécialisé, chef de l'Unité d'Animation Technique (UAT) dans la commune de Boulmiougou, vulgarisatrice des activités de maraîchage, il est ressorti que la formation des maraîchers par l'agent technique d'agriculture porte essentiellement sur les techniques culturales au détriment de l'hygiène des légumes : "Jusqu'à présent, on ne nous a pas donné ça (thème sur l'hygiène des légumes) comme thème de 
formation. C'est quand tu vois que ça ne va pas, tu vois quelqu'un qui arrache son pied de laitue, qui secoue dans un puisard, qui commence à manger ou bien la carotte, bon, tu ne peux que lui dire bon, il y a les produits chimiques, même dans l'eau de puits, on retrouve les produits chimiques".

Par ailleurs, après analyse de l'entretien fait avec le chargé des affaires économiques et sociales de la Mairie de Boulmiougou, un des chefs de section de ce service, nous remarquons que très peu d'initiaves sont prises par la mairie pour protéger les consommateurs de légumes crus contre les risques d'épidémies de choléra. En effet, à la question de savoir quelle relation la Mairie entretient avec les maraîchers de ce site, l'enquêté répond : "Bon, je n'ai pas grande connaissance, ce n'est qu'en 2007 qu'un arrêté signé par la Mairie Centrale de Ouagadougou, m'a nommé comme membre représentant de madame le Maire dans un projet pilote pour l'intensification de la maraîcheculture. Donc, c'est à ce titre, j'ai appris à connaître les maraîchers, ce qu'ils font. Voilà ! ». En outre, à la question de savoir quel projet a intervenu sur le site dernièrement ? II informe : "II y a des questions auxquelles moi je ne peux même pas répondre parce qu'avec les maraîchers, on ne s'est pas entendu, moi je les ai quitté. Je ne pars plus là-bas" ".

Cette information montre que les maraîchers ne sont pas suivis, ne sont pas visités.

Aussi, après analyse des opinions et déclarations faites par le Directeur du contrôle des aliments et de la nutrition appliquée, nous retenons que le Laboratoire National de Santé Publique (LNSP) ne mène pas des actions quant à la prévention de la maladie du choléra.

Pour l'agent interviewé, faire des analyses pour prévenir la maladie du choléra n'est pas nécessaire car cette maladie a une période d'incubation très courte. Aussi, a-t-il soutenu, même si des analyses devraient être faites pour les légumes crus, c'est sur la table du consommateur et jamais des légumes qui se trouvent encore sur le site de maraîchage ou sur le marché et destinés à la vente : "Dans aucun labo au monde on ne cherche systématiquement la bactérie du choléra parce que ce n'est pas quelque chose qui est courant par rapport aux autres microorganismes ... L'aspect microbio là, l'analyse se fait uniquement au point de distribution finale, à la table du consommateur. Voilà ! On ne peut pas incriminer celui qui a produit, celui qui a vendu au marché. C'est celui qui a servi la salade prête à être consommée-là qui est responsable de la situation", justifie le Directeur du contrôle des aliments et de la nutrition appliquée du LNSP, oubliant que beaucoup sont ceux qui consomment les légumes crus sans assaisonner sur les sites de maraîchage et dans les marchés. Pour cette raison, même quand une institution quelconque demande au LNSP de faire des analyses dont les prestations seront payées par ladite institution, le LNSP prend la décision de ne pas inclure la bactérie du choléra dans leurs analyses de laboratoire. Prenant l'exemple sur le Centre National des Fuvres Universitaires (CENOU) qui bénéficie des prestations du LNSP, l'agent interrogé avoue : "C'est nous qui déterminons nos paramètres, le CENOU ne nous impose pas de paramètre. Voilà ! Tout ce qu'on fait, il paye. Ça, il n'y a pas de débat sur ça. Voilà ! La bactérie responsable du choléra, on ne la recherche pas dans ces produits-là parce que c'est une bactérie extrêmement rare. Voilà ! Ce qui fait qu'on ne la recherche pas ". Cette intervention prouve que la maladie du choléra est négligée.

Il souligne aussi le manque de moyens financiers qui est un handicap à l'analyse des légumes crus prêts à être consommés : "Ces salades-là ne sont pas contrôlées parce que c'est une question de prise en charge des frais d'analyses; peut-être vous allez trouver quelqu'un qui vend tout au plus 20 plats par jour. Vous allez lui demander de payer combien ? L'analyse des salmonelles à elles seules coûte $17000 \mathrm{~F}$ CFA et Eichericia coli coûte I 1000 F CFA. Ça fait en tout 28000 F CFA ... Nous n'entretenons pas de relation directe avec les maraîchers parce que d'abord c'est des activités qui sont menées dans de petits lopins de terre. En général, ce sont des exploitations familiales et ici nos activités, nos prestations sont payantes. Aujourd'hui si on se déporte chez un maraîcherculteur qui ne produit qu'un petit panier de légumes par jour alors que les analyses coûtent assez chères, alors que l'intéressée même, toute sa marchandise ne vaut même pas peut être 2000 F CFA, vous allez demander à une dame comme ça de payer ?Si on s'en tient aux salmonelles et à Eïchericia coli, c'est 28000 F CFA par analyse. Vous voyez, c'est difficile ".

De même, suite à l'entretien réalisé avec le chef de service de l'hygiène alimentaire et de la salubrité, à la Direction de l'action sanitaire de la commune de Ouagadougou, il ressort que le Service d'hygiène ne mène qu'occasionnellement des actions en matière d'hygiène des légumes crus : "II va s'en dire que compte tenu du fait que notre action ne porte pas uniquement sur les légumes et les produits crus, on ne peut pas percevoir particulièrement notre action en permanence sur ces produits-là comme si nous avions une unité qui était chargé uniquement du contrôle des produits crus .... Dans tous les secteurs de la ville de Ouagadougou, nous faisons le contrôle de tout ce qui est produit alimentaire et il va s'en dire que quand on fait la balance, l'action concrète sur les produits crus, donc des légumes est faible ... l'inspection porte également sur l'assainissement et la 
sécurité même à l'intérieur du marché. Ça porte sur les compartiments du marché, donc, y compris la zone des revendeuses de légumes. Nous constatons des anomalies, nous leur faisons les remarques et séances tenante, nous faisons une petite sensibilisation en leur montrant comment il faut faire. En montrant à l'individu bien sûr avec ceux qui sont à côté qui profitent également. On leur montre comment il faut faire pour garantir de bonnes conditions d'hygiène au niveau de ces étals-là).

En outre, les actions sur les légumes crus sont menées lors des épidémies du choléra et après ces épidémies, ces actions aussi prennent fin par manque de moyens économiques : "En 2005, nous avons eu à faire une opération de contrôle au niveau du marché et des abords de l'hôpital. Le petit marché de Paspanga (quartier de Ouagadougou), nous l'avons inspecté et ce jour-là, nous avons eu effectivement à ramasser des étals de vendeuses qui avaient même posé leurs légumes à même le sol. C'est pour dire que ça, c'est une action concrète qui a été menée et qui portait sur les fruits et légumes... Comme c'était pendant l'épidémie de choléra, il fallait passer à la répression ... Nous avons eu à faire quand même des opérations à ce niveau et essentiellement des opérations de sensibilisation, des opérations également de contrôle et nous avons eu à faire des actions d'appui, d'assainissement à ce niveau-là à une période par exemple critique, au temps du choléra. Voilà, nous avons eu à désinfecter les eaux des puits. Tous les sites de maraîchages qui sont aux alentours des barrages là, nous avons eu à les (barrages) désinfecter... Sur le site de maraîchage de Boulmiougou précisément, après 2005 nous n'avons pas fait une autre action concrète... Mais au moment du choléra, là, il y avait des spots télés, il y avait des émissions Radio, c'était vraiment un matraquage médiatique et là, nous avons effectivement fait beaucoup d'interventions à la Radio, à la Télé, à travers les affiches mêmes et des rencontres au niveau des marchés tout ça pour passer l'information. Quand le choléra disparait, les moyens qui devaient permettre de mener les actions disparaissent égalements.

Quant au Secrétaire permanent de la Ligue des consommateurs du Burkina, il soutient que les actions de la Ligue des consommateurs vont généralement à l'endroit de ses membres et qu'à l'égard des maraîchers, c'est l'Etat qui doit s'en charger : «Nous organisons des formations à l'endroit de nos membres surtout parce que nous sommes là à cause des adhérents. Donc nos actions vont surtout en direction de nos membres pour les sensibiliser par rapport à un domaine bien précis ... Nous sommes mal placé pour aller dire à un jardinier voilà, il faut cultiver ici, il faut faire ceci, il faut faire cela... Aux alentours de ces zones polluantes, nous estimons que l'Etat doit prendre toutes les dispositions pour que dans ces zones-là, on ne pratique pas le maraîchage ".

Pour résumer, suite à la rencontre d'avec ces différentes institutions, nous pouvons dire que les mesures prises pour garantir la salubrité des légumes crus ne sont pas suffisantes. D'une part, l'épidémie du choléra est négligée. D'autre part, des difficultés d'ordre financier, matériel semblent être les handicaps majeurs dans la réalisation des objectifs de ces différentes structures œuvrant dans le domaine de l'Hygiène alimentaire. Le pouvoir de ces institutions semble plutôt être fictif, passif. A cet effet, elles n'arrivent plus à s'imposer. Elles semblent se contenter de sensibiliser de temps en temps la population en période d'épidémie de choléra.

Alors, les consommateurs courent d'énormes risques à consommer les légumes crus du site de maraîchage de Boulmiougou et du quartier de Pissy étant donné qu'aucune analyse n'est faite, alors que la bactérie du choléra n'est pas visible à l'œil nu.

\section{Solutions perçues par les enquêtés}

Les solutions proposées par la majeure partie des interviewés vont à l'égard de l'Etat qui doit mettre l'accent sur la sensibilisation de la population et la formation des maraîchers. C'est ce que stipule cette revendeuse : «ll faut que l'Etat sensibilise les gens. Tu peux laver les légumes de ta manière et ne pas pouvoir tuer les bactéries de choléra. Par contre, si l'Etat nous sensibilise, tu sais que pour pouvoir tuer les bactéries, il faut laver de telle manière " (revendeuse de 45 ans, non instruite, mariée, mossi).

Un maraîcher suggère : " II faut une sensibilisation et une formation des maraîchers par l'Etat parce que nous ne voulons pas par ignorance, produire des légumes qui vont provoquer des maladies pour les consommateurs. Nous voulons donc une sensibilisation " (maraîcher de 20 ans, niveau d'instruction primaire (CMI), mossi, marié).

Une ménagère renchérit : "Notre suggestion, les autorités eux-mêmes peuvent sortir pour contrôler les aliments, la nourriture qui est prête à être consommée... Si elles sortent, elles vont trouver des gens qui vendent les légumes pourris. Souvent c'est des enfants. Est-ce que l'enfant connait quelque chose? Si nous trouvons cela, il y a certaines personnes qui n'ont aucune idée de l'hygiène et qui vont apprendre. D'autres connaissent, mais s'ils vont bénéficier encore des conseils, cela peut beaucoup les aiden (ménagère de 45 ans, non instruite, mossi, mariée).

Certaines enquêtées reconnaissent que la population a une part de responsabilité concernant la consommation de légumes crus insalubres et par 
conséquent, formulent des revendications à l'égard de l'Etat et propose des solutions que la population peut elle-même adopter. Le discours de cette ménagère est illustratif de cet état de fait : " Comme suggestion, je demande aux revendeuses de laver les légumes avant de revendre. Je demande aux consommateurs de bien laver aussi avant d'assaisonner, laver 3 à 4 fois. Le Gouvernement, je lui demande de mettre des gens sur place pour sensibiliser la population» (ménagère de 30 ans, non instruite, mossi, mariée).

Les solutions proposées par les autorités intervenant dans le domaine de l'Hygiène alimentaire sont la formation des maraîchers et la sensibilisation de la population sur l'hygiène des légumes :

" ...l'Etat, surtout le Ministère de l'agriculture doit essayer de former les producteurs sur l'hygiène des légumes consommés crus. II faut le faire un peu partout. Il faut commencer par le site, ceux qui produisent. La sensibilisation doit aller du producteur au consommateur. Il y a des services d'hygiène qui peuvent s'en occuper ou dans le cas contraire, si l'agent d'encadrement est formé en ce sens, je me dis que ça peut apporter un plus à l'hygiène sur le site " (agent technique d'agriculture spécialisé intervenant sur le site de maraîchage de Boulmiougou).

" Moi, je pense qu'il faut donner plus de moyens aux producteurs. II faut les former parce que c'est des gens qui sont laissés à eux-mêmes" (chargé des affaires économiques et sociales de la Mairie de Boulmiougou).

"Ce que je peux proposer, c'est la mise en place d'un plan d'étude sanitaire qui consiste à maitriser les bonnes pratiques d'hygiène dans le milieu, dans l'environnement dans lequel nous vivons... laver les mains avant de manger, bien laver les ustensiles... nettoyer et désinfecter les légumes crus avant de les consommer. La solution c'est cela. Sinon sur le site de maraîchage, on n'y peut rien... Au Ministère de la santé ou bien l'Etat, C'est de donner les moyens aux structures qui sont chargées de cette prévention là de faire leur travail. Et mettre l'accent aussi sur la sensibilisation, les émissions Radio, Télé... Mettre à notre disposition un certain nombre de moyens pour qu'on puisse faire des études... Ces résultats là peuvent être publiés " (Directeur du contrôle des aliments et de la nutrition appliquée du LNSP).

«... Mettre en cuvre un certains nombres d'actions à savoir la formation même au niveau des maraîchecultures... il va falloir chlorer les eaux des puits, étaler les légumes et les fruits sur des surfaces élevées et bien protégées" (chef de service de I'hygiène alimentaire et de la salubrité, Direction de l'action sanitaire de la commune de Ouagadougou).

"Le laboratoire malheureusement n'a pas de moyens. Nous demandons à l'Etat de donner des moyens (au Laboratoire National de Santé Publique), donner les moyens à cette structure de répondre à son rôle. Il faut une sensibilisation de la population de bouche à oreille. De la part des autorités... Il faut que les populations connaissent ce qu'on appelle le choléra, comment ça se contracte» (Secrétaire permanent de la Ligue des consommateurs du Burkina).

\section{DISCUSSION}

\section{Limite de l'étude}

Le présent travail de recherche a concerné à la fois les producteurs de légumes crus, les revendeuses de légumes et les consommateurs. Ce qui ne permet pas d'explorer en profondeur la notion du propre et du sale comme cela aurait été possible dans le cadre d'une enquête adressée à une seule catégorie de personnes (soit les maraîchers, ou les revendeuses de légumes crus ou encore les traiteurs de ces légumes). II serait souhaitable que d'autres études prenant en compte une seule catégorie d'acteurs soit menées sur ce sujet dans le quartier de Pissy et sur le site de maraîchage de Boulmiougou pour approfondir les connaissances des acteurs sur les notions de "propre " et de "sale " dans le contexte du maraîchage.

En outre, les déclarations des maraîchers, des revendeuses de légumes crus et des consommateurs lors de l'enquête peuvent être affectées par des oublis mais aussi par le souci de donner des informations conformes aux messages sanitaires hygiénistes contrairement aux réalités couramment vécues. Par conséquent, il serait difficile d'évaluer la prévalence réelle de leurs pratiques sur les risques d'épidémie de choléra liés à la commercialisation et à la consommation des légumes crus.

Connaissances liées au mode de transmission de la maladie du choléra

II est ressorti de notre étude que la majorité des personnes interrogées ignore les déterminants du choléra. Des risques de contamination lors de la production des légumes à cause de la mauvaise qualité de l'eau d'arrosage, lors de la commercialisation de ces légumes et lors de leur traitement avant consommation compte tenu de la non-utilisation de produits désinfectant ou de l'utilisation de désinfectants inappropriés ont été relevés.

Cette ignorance du mode de transmission de la maladie du choléra quasi-partagée par nos enquêtés n'est pas spécifique à cette enquête. Nos résultats sont similaires à ceux de Kassié (2007 : 67) qui dans son étude conduite sur les caractéristiques sociospatiales du choléra en 2005 à Ouagadougou avait attesté que l'ignorance du mode de transmission de la 
maladie avait pu contribuer à la contamination d'autres malades. L'auteur soutien que les piqûres de moustiques ont été évoquées par $11 \%$ des enquêtés comme étant responsables de la transmission du choléra. II semble même que sa prévention puisse résider dans l'utilisation de moustiquaires imprégnées. Par ailleurs, il assure que la connaissance de germes pouvant être responsables de maladies semble échapper à nombre des enquêtés qui réfutent l'idée que l'on puisse contracter la maladie en buvant de l'eau.

Perception relative à l'eau utilisée pour le maraîchage Notre étude a montré que sur II maraîchers enquêtés, 10 estiment que les lieux de productions des légumes sont salubres et par conséquent, il n'y a pas de possibilité de contracter le choléra sur le site de maraîchage. En plus, la totalité de ces maraîchers $(\mathrm{II} / \mathrm{II})$ pense que l'eau utilisée pour arroser les légumes est propre et sans aucun risque de contamination. Beaucoup d'entre eux préfèrent utiliser l'eau de puits ou du barrage sous prétexte que ces deux types d'eau font mieux développer les légumes comparativement à l'eau du forage.

Nos résultats rejoignent ceux de Ouédraogo et al., (2007 : I). En effet, ces auteurs dans leur étude conduite sur la Gestion des risques en agriculture urbaine irriguée et consentement à payer pour une amélioration de la qualité de l'eau pour le maraîchage dans les villes de Ouagadougou et de Bobo-Dioulasso au Burkina Faso soutenaient que moins d'un sixième des producteurs ont pleinement conscience des risques sanitaires liés à l'utilisation des eaux usées.

Cette perception que les maraîchers ont de l'eau disponible sur les sites de maraîchage semble être un frein à l'adoption de stratégies visant à améliorer la qualité de l'eau. Comme le disait Ouédraogo (2008: 34) dans son travail de recherche réalisé sur les facteurs de vulnérabilité et stratégies d'adaptation aux risques des maraîchers urbains et périurbains dans les villes de Ouahigouya et de Koudougou, plutôt que d'améliorer la qualité de l'eau, l'objectif majeur est d'augmenter la capacité des puisards à mobiliser l'eau.

Insuffisance d'actions menées dans la lutte contre l'épidémie du choléra

Les résultats de nos entretiens avec les agents des institutions travaillant dans le domaine de l'hygiène ont relevé que les actions menées pour préserver la santé des populations contre l'épidémie du choléra ne sont pas suffisantes. La maladie du choléra semble être négligée en ce sens qu'il n'y a pas suffisamment de sensibilisation concernant l'hygiène des légumes consommés crus dans le quartier de Pissy et compte tenu du fait que les maraîchers du site de maraîchage de Boulmiougou ne sont pas formés à cet effet. La formation de ces derniers par l'agent technique d'agriculture porte plutôt sur les techniques culturales. Nos résultats sont similaires à ceux de Zeba (2003 : 55 et 76) qui dans son étude menée sur les risques liés au travail des maraîchers dans la ville de Ouagadougou, site de Boulmiougou, soutenait qu' " il n'existe pas de personne formée pour la sécurité et l'hygiène sur le site ". Cette auteure avait mentionné que malgré l'insistance des maraîchers pour impliquer les autorités municipales et sanitaires, aucune descente sur le terrain des agents concernés ne s'était effectuée.

Risques de contaminations liés au faible niveau d'éducation de la population enquêtée

Nos enquêtes de terrain ont relevé qu'il y a un lien entre le niveau d'instruction et les pratiques des acteurs concernant la propreté des légumes crus. En effet, même si les normes au niveau des pratiques de désinfection ne sont pas respectées, parmi les quinze préparateurs de légumes crus interrogées (Ménagères, restaurateurs et traiteurs de légumes crus dans la rue) dont six instruits et neuf illettrés, toutes les personnes instruites (6/6), désinfectent au moins la laitue avec le permanganate ou l'eau de javel. Ainsi, ceux qui désinfectent les légumes plus ou moins convenablement avec l'eau de javel ou du permanganate sont majoritairement instruits.

Sur ce plan, Diolompo (2009) dans son étude conduite sur l'alimentation de rue à Bobo-Dioulasso au Burkina Faso avait remarqué cet état de fait. II avait montré que $59,25 \%$ de consommateurs étaient analphabètes, leur niveau d'instruction étant faible, les mesures d'hygiène nécessaires pour la prévention de certaines maladies par conséquent pouvaient être méconnues.

Risques de contaminations liés à la situation économique de la population enquêtée

En rappel, lors des entretiens individuels approfondis, le niveau du pouvoir d'achat du consommateur était déterminant pour la consommation de légumes crus salubres. La quasi-totalité de nos enquêtés a décelé un seul facteur le plus souvent pour expliquer la consommation de légumes crus insalubres. D'une part, la pauvreté a été développée par plusieurs enquêtées pour justifier l'achat des légumes crus pourris. D'autre part, bien que reconnaissant l'inefficacité de certains produits dans la désinfection des légumes crus, des intervenants déclarent être obligés de les utiliser parce que ne disposant pas d'argent pour acheter les désinfectants habilités à jouer ce rôle.

Nos résultats contrastent avec ceux de Bompangue Nkoko (2009 : 203) dans son étude sur la dynamique 
des épidémies de choléra dans la région des grands lacs Africains en République Démographique du Congo. Cet auteur réfute l'idée selon laquelle le choléra soit lié à la pauvreté sans autres explications. Pour lui, le choléra n'est ni une maladie du "pauvre ", ni une maladie de la pauvreté.

\section{Conclusion}

L'étude a permis d'avoir un aperçu global sur les risques de choléra liés aux pratiques des maraîchers du site de Boulmiougou, des revendeuses du marché de Pissy et des consommateurs de ce quartier. Après analyse des résultats des entretiens approfondis réalisés auprès des différents acteurs et de l'observation des pratiques, il ressort que :

Les différents acteurs (producteurs, revendeuses, consommateurs des légumes crus) ont des pratiques à risque liés à leurs perceptions de l'eau utilisée, de la notion de saleté et de leur ignorance des modes de transmission de la maladie du choléra.

Les pratiques à risques des consommateurs sont déterminées par la situation socio-économique des acteurs du maraîchage, le faible niveau d'éducation de la population et l'inobservation des règles d'hygiène susceptibles de transmettre la bactérie du choléra aux consommateurs de ces légumes crus.

Les stratégies de réduction de risque sont entre autres la formation des maraîchers par l'Etat burkinabè, l'information et la sensibilisation de la population sur les bonnes pratiques d'hygiène.

Le niveau critique d'insalubrité des légumes crus est dû en partie à l'insuffisance d'informations et de formations sur les effets de celui-ci. Ainsi, pour un quartier périphérique comme celui de Pissy, la sensibilisation, l'information de la population, ainsi que la formation des maraîchers de Boulmiougou apparait nécessaire pour une lutte efficace contre le choléra. Une telle lutte devrait impliquer davantage aussi bien les populations elles-mêmes, les structures communales et étatiques, les partenaires du privé, les associations et ONG qui se sont engagées. Ceux-ci sont invités à mettre l'accent sur le contrôle des eaux usées au laboratoire, notamment sur les pollutions bactériologiques.

Force est de constater que de nos jours, les populations des villes du Burkina Faso s'intéressent de plus en plus à la culture et à la consommation des légumes crus. Par conséquent, notre étude ne saurait à elle seule épuiser le contenu de notre question de départ. L'insalubrité des légumes crus constitue un phénomène crucial dans le quartier de Pissy et dans le site de maraîchage de Boulmiougou, et mérite que plusieurs chercheurs s'y intéressent davantage.

Par ailleurs, ce travail de recherche s'est limité au site de maraîchage de Boulmiougou, le seul site de
Ouagadougou reconnu d'utilité publique par l'Etat Burkinabè. Des études portant sur d'autres sites de maraîchage de Ouagadougou moins reluisants que celui de Boulmiougou ainsi que d'autres quartiers de la dite ville devraient être envisagées.

\section{REFERENCES BIBLIOGRAPHIQUES}

Bagbila, Joël. (2007). Utilisation des déchets urbains en Maraîcheculture: Risques de contamination des végétaux par les métaux lourds. Mémoire de fin d'études, Institut du Développement Rural (IDR) / Université Polytechnique de Bobo-Dioulasso (UPB), 69 p. + annexes

Bompangue Nkoko, Didier. (2009). Dynamique des épidémies de choléra dans la région des grands lacs Africains: cas de la République Démographique du Congo. Thèse de doctorat en Sciences de la vie et de la santé, Université de Franche-Comte, $220 p+$ Annexes

Cissé, Gueladio. (1997). Impact Sanitaire de l'utilisation d'eaux polluées en Agriculture urbaine : cas du maraîchage à Ouagadougou (BF). Thèse de Doctorat es sciences techniques $n^{\circ}$ 1639. Lausanne : EPFL, 267 p. + annexes

Centre collaborant de l'OMS. (200I). Un fléau qui fait des ravages chaque année. Recueil d'articles $\mathrm{N}^{\circ}$ I, info CREPA $\mathrm{N}^{\circ}$ 9-Juillet-Août-Septembre 1995, 51 I p.

Diolompo, Soyoro. (2009). Etude des facteurs influençant la précarité de l'hygiène des aliments de la rue : cas du marché de Nieneta. Commune de Bobo-Dioulasso, Mémoire de fin d'études, Ecole Nationale de Santé Publique (ENSP), 92 p.

Direction des Etudes et de la Planification (DEP). (20/2). Analyse synthétique des résultats de l'énumération de la population de Ouagadougou de 20I2. Ouagadougou, $20 \mathrm{p}$.

Direction Générale de la Promotion de l'Economie Rurale (DGPER). (20I I). Rapport d'analyse du module maraîchage. Ouagadougou, Burkina Faso, rapport, $215 \mathrm{p}$.

Drabo, Koiné Maxime, et al. (2008). Caractéristiques de l'alimentation de rue dans la ville de Bobo-Dioulasso, Burkina Faso. Santé publique, (3267), 36-40

Organisation des Nations Unies pour l'alimentation et l'agriculture (FAO). (20I0). L'état de l'insécurité alimentaire dans le monde 2010: Combattre l'insécurité alimentaire lors des crises prolongées. Rome, Italie, 2 p.

Organisation des Nations Unies pour l'alimentation et l'agriculture (FAO). (2005). La situation mondiale de l'alimentation et de l'agriculture. Le commerce agricole et la paurreté : le commerce peut-il être au service des pauvres?, Rome, $2 / 2$ p. 
Institut National de la Statistique et de la Démographie (INSD). (20I5). Profil de pauvreté et d'inégalités. Enquête Multisectorielle Continue (EMC) 2014, Ouagadougou, Burkina Faso, rapport, $90 \mathrm{p}$.

Institut National de la statistique et de la démographie (INSD). (2006). Résultat définitifs du recensement général de la population et de I'habitat. Recensement Ggénéral de la Population et de l'Habitat (RGPH), Ouagadougou, Burkina Faso, rapport, $55 \mathrm{p}$.

Kabore, Pauline. (2005). Citoyenneté et foncier urbain: cas de l'agriculture urbaine dans l'arrondissement de Boulmiougou (BF). Mémoire de maîtrise de Sociologie, Ouagadougou, Université de Ouagadougou, $145 \mathrm{p}+$ annexes

Kassié, Daouda. (2007). Caractéristiques sociospatiales du Choléra en 2005 à Ouagadougou : cas des secteurs 23 et 24 . Département de Géographie, mémoire de maîtrise, Ouagadougou, Université de Ouagadougou, 84 p. + annexes

Direction de l'hygiène publique et de l'éducation pour la santé du Ministère de la santé (2003). Guide Pratique: Mesures d'hygiène pour prévenir et lutter contre le choléra. Ouagadougou, Burkina Faso, rapport, $29 \mathrm{p}$.

Olivier de Sardan, Jean.-Pierre. (2005). La rigueur du qualitatif: Les contraintes empiriques de l'interprétation socio-anthropologique. Louvain-laNeuve : Academia Bruylant, 2005. 365 p.

Ouédraogo, Abdoulaye. (2008). Facteurs de vulnérabilité et stratégies d'adaptation aux risques des maraîchers urbains et périurbains dans les villes de Ouahigouya et de Koudougou. Mémoire de maîtrise, Institut du Développement Rural (IDR) / Université Polytechnique de BoboDioulasso (UPB), 58 p. + annexe

Ouédraogo, Dénis, et al. (2007). Gestion des risques en agriculture urbaine irriguée et , Ouagadougou, 89 p. + annexes consentement à payer pour une amélioration de la qualité de l'eau pour le maraîchage dans les villes de Ouagadougou et de Bobo-Dioulasso au Burkina. Ouagadougou, Burkina Faso, rapport, I5 p.

Pangni, Tiburce. Claudaire. (2003). Production maraîchère dans les provinces du Seno et du Yagha: Analyse et perspectives. Mémoire de fin de cycle, Institut du Développement Rural (IDR) / Université Polytechnique de Bobo-Dioulasso (UPB), 72 p. + annexes

Peretti-Watel, Patrick. (2004). Du recours au paradigme épidémiologique pour l'étude des conduites à risque. Revue française de sociologie (Vol 45), 103-132.

Quivy, Raymond, et Van Campenhoudt, Luc. (1995). Manuel de recherche en sciences Sociales. Paris : Dunod, $287 \mathrm{p}$.

Sadikou, Oumarou. (2004). Epuration des eaux usées domestiques par procédé UASBValorisation des sous-produits de l'épuration dans la production maraîchère. Ouagadougou, Burkina Faso, rapport, $40 \mathrm{p}+$ annexes

Thiombiano, Boundia. (2008). Analyse de la contribution des cultures de saison sèche à la lutte contre la pauvreté au Burkina. Mémoire de fin de cycle, IDR / UPB, 55 p. + annexes

Tougma, Téné. Arlette. (2007). Déterminants de la durabilité des pratiques d'irrigation dans les systèmes de productions maraîchères urbains et périurbains au Burkina: Analyse de la situation à Bobo-Dioulasso et Ouagadougou. Mémoire de maîtrise, IDR / UPB, 68 p. + annexes

Zeba, Marguerite.-Marie. (2003). Les risques liés au travail des maraîchers dans la ville de Ouagadougou site de Boulmiougou. Mémoire de fin d'études,

ihttp://www. troublenutritionnel.wikibis.com/cholera.php, consulté le 16 Février 2016 